\title{
Assessing Relative Stressors and Mental Disorders among Canadian Provincial Correctional Workers
}

\author{
Katy Konyk $^{1} \mathbb{D}$, Rosemary Ricciardelli ${ }^{2, *(\mathbb{D}}$, Tamara Taillieu ${ }^{3} \mathbb{D}$, Tracie O. Afifi ${ }^{3} \mathbb{D}$, Dianne Groll ${ }^{4} \mathbb{D}$ \\ and R. Nicholas Carleton 5 (i)
}

1 School of Social Work, McGill University, Montreal, QC H3A 1E3, Canada; katy.konyk@mail.mcgill.ca

2 Department of Sociology, Memorial University of Newfoundland, St. John's, NL A1C 5S7, Canada

3 Rady Faculty of Health Sciences, University of Manitoba, Winnipeg, MB R3E 0W3, Canada; tamara.taillieu@umanitoba.ca (T.T.); tracie.afifi@umanitoba.ca (T.O.A.)

4 Departments of Psychiatry and Psychology, Queen's University, Kingston, ON K7L 3N6, Canada; grolld@queensu.ca

5 Department of Psychology, Anxiety and Illness Behaviours Laboratory, University of Regina, Regina, SK S4S 0A2, Canada; nick.carleton@uregina.ca

* Correspondence: rricciardell@mun.ca; Tel.: +1-709-864-7446

check for updates

Citation: Konyk, K.; Ricciardelli, R.; Taillieu, T.; Afifi, T.O.; Groll, D.; Carleton, R.N. Assessing Relative Stressors and Mental Disorders among Canadian Provincial Correctional Workers. Int. J. Environ. Res. Public Health 2021, 18, 10018. https://doi.org/10.3390/ ijerph181910018

Academic Editor: Paul B. Tchounwou

Received: 13 August 2021

Accepted: 14 September 2021

Published: 23 September 2021

Publisher's Note: MDPI stays neutral with regard to jurisdictional claims in published maps and institutional affiliations.

Copyright: (c) 2021 by the authors. Licensee MDPI, Basel, Switzerland. This article is an open access article distributed under the terms and conditions of the Creative Commons Attribution (CC BY) license (https:// creativecommons.org/licenses/by/ $4.0 /)$.

\begin{abstract}
In the current study, we quantified the mean stress levels of 43 occupational stressors for 868 Correctional Workers (CWs) and analyzed the relationships between occupational stressors, exposure to potentially psychologically traumatic events (PPTEs), and mental health disorders. Our findings emphasize the importance of the occupational environment in relation to $\mathrm{CW}$ mental health and indicate that occupational stressors (e.g., staff shortages, inconsistent leadership style, bureaucratic red tape) are more salient contributors to CW mental health than exposure to PPTEs. Finding strategies to ameliorate staff shortages, improve leadership style and communication, and support CWs to maintain physical, mental, and social well-being would be interventions tied to significant organizational and operational stressors within the current study.
\end{abstract}

Keywords: occupational stressors; correctional workers; potentially psychologically traumatic events; mental health disorders; PTSD

\section{Introduction}

Correctional workers (CWs) perform physically and emotionally demanding jobs in prison or community environments that remain largely hidden from the public [1,2]. CWs include all employees within all branches of correctional services, from community to administration to institutional, while correctional officers (COs) are the uniformed workers responsible for the care, custody, and control of prisoners [3,4]. COs are the first responders within prisons [5] and provide multidimensional services to balance the safety, security, and rehabilitation needs of prisoners [4]. Researchers are increasingly drawing public attention to the risks and potential mental health consequences associated with working in institutional (e.g., prisons) [6] or in community correctional services (e.g., parole offices) [7].

The conceptualization and measurement of employee psychological well-being varies across studies with CWs. Research on CW psychological well-being commonly operationalizes the outcome as job stress, the "psychological strain leading to job-related hardness, tension, anxiety, frustration, and worry arising from work" [8] (p. 23); however, recent research is increasingly focused on symptoms of Posttraumatic Stress Disorder (PTSD), Major Depressive Disorder (MDD), and Generalized Anxiety Disorder (GAD) as common psychological outcomes associated with exposure to potentially psychologically traumatic events (PPTEs) [9] within correctional services work [6,10-12]. Early research demonstrates that the Canadian prevalence of mental health disorders among CWs is higher than other public safety personnel (PSP) groups $[13,14]$. The available evidence suggests that occupational stressors (i.e., operational stressors related to job content; organizational stressors 
related to job context) may be as impactful on PSP psychological well-being as exposure to PPTEs [15], but results specific to CWs remain sparse (for a discussion of organizational and operational stress with the policing context see Duxbury and Higgins, 2001; 2015). Thus, in the current article, we analyze the relationship between 43 occupational stressors and mental health disorders amongst Ontario CWs. We hypothesize that occupational stressors have the potential to be just as detrimental to CW stress and mental health as PPTEs. We begin the article by reviewing research on stressors in the correctional environment followed by empirical knowledge on CW psychological well-being. In the results, we elucidate the various degrees to which occupational stressors contribute to $\mathrm{CW}$ stress levels and mental health disorders. We conclude the paper with a discussion of the results and implications for interventions tied to employee psychological well-being.

\subsection{Correctional Worker Occupational Stressors and Exposure to PPTEs}

The research on occupational stressors, prevalence of PPTE exposures, and relationships between PPTEs and mental health among CWs and other PSP is growing [15-18]. The recent growth has been facilitated by calls to action from the Government of Canada $[19,20]$. The associations between occupational stressors, job stress, and burnout have received substantial scholarly attention [3]; however, past research has rarely examined how occupational stressors impact mental health and there is almost no research specific to CWs.

Previous researchers have implicated role problems, supervision, work-family conflict, fear of victimization, and exposure to workplace violence as commonly analyzed occupational stressors [21]; nevertheless, the conceptualization and measurement of independent stressor variables, as well as the number of variables included for analyses, have varied considerably in datasets from CWs. The absent data has obfuscated attempts to understand the relative impact of stressors on mental health [22], despite the emphasized need for clarity [21].

Correctional scholars have differentiated role conflict, role ambiguity, and role overload [21]. Role conflict can result from the duality inherent in expectations that employees will meet both the security needs of an institution and the rehabilitation needs of prisoners [23]. Role ambiguity refers to uncertainty regarding how to perform one's role or what tasks the role encompasses [21,23]. Role overload captures the tension and stress that can arise from too high a workload [21]. All three are positively associated with job stress and burnout [22,24-26] and with PTSD [12].

The quality of supervision and guidance provided to CWs can dramatically influence experiences of job stress [21], with certain aspects of supervision being more significant than others [26]. Supervision is less frequently examined as a variable impacting the development of mental disorders. In one of the few studies available, reported positive supervisory relationships appear significantly correlated with lower self-reported symptoms of PTSD [12].

Overlapping roles and responsibilities regarding work and family can also dramatically influence experiences of stress [21]. Strain and behavior-based conflicts appear to be the most salient domains of work-family conflict correlating with job stress for CWs [27-29]. Work-family conflict also appears associated with elevated prevalence of depression for COs [30] and the emotional exhaustion component of burnout amongst female correctional staff [31]. The regimented nature of carceral work environments may contribute to challenges transitioning between work and family [27]. For example, the rush of adrenaline and associated negative emotions that can occur when using physical force to intervene in prisoner conflict may linger beyond the work shift thereby contributing to problems at home [32].

The consistent potential need to respond to violence in correctional environments has led to research on CW perceptions of danger in the workplace and job stress. Most studies evidence a significant and positive association between perceptions of threat, perceived job stress [24,26,33-35], and burnout [36]. The results underscore that regular perceptions of 
threat experienced by CWs are associated with personal safety concerns and job stress [23], but the specific mechanisms of how their perceptions increase job stress remain unexplored.

COs will likely experience or witness violence during their career $[37,38]$ and this expectation may influence perceptions of employee safety. The Union of Canadian Correctional Officers (UCCO-SACC-CSN) suggests that $98 \%$ of COs will be exposed to a PPTE at some point during their career [39]. In the United States (US), between 1999 and 2008 there were 113 work-related fatalities (e.g., fatal assaults, suicide) amongst COs and 125,200 nonfatal work-related injuries that received treatment in an emergency department [40]. A nationwide survey of Canadian PSP established the prevalence of lifetime PPTE exposures [10] using a slightly modified version of The Life Events Checklist for the DSM-5 [41,42]. The most common PPTEs for Canadian CWs are physical assault $(88.7 \%)$, sudden violent death $(85.6 \%)$, sudden accidental death $(80.6 \%)$, assault with a weapon $(78.8 \%)$, and life-threatening illness or injury (77.9\%) [10]. CWs in the United States experience an average of 28 exposures to violence, injury, or death events [43]. COs at a provincial jail in Atlantic Canada associated occupational stressors with negative physical or mental health outcomes related to one or more workplace PPTEs [38]. COs described strategies of 'emotional distancing' from prisoner suffering and normalization of violence to help cope with regular exposure to PPTEs. Ricciardelli and Power (2020) conclude that CO mental health suffers at the intersection between exposure to violence and poor organizational response.

PPTE exposure is a defining feature of PTSD [44]; however, individuals may manifest other forms of psychological distress post exposure to a PPTE, including depression and anxiety [45]. PPTE exposures have been significantly associated with increased risks for PTSD and other posttraumatic stress injuries (PTSI; e.g., MDD, GAD) among diverse Canadian PSP including CWs [10]. Despite conceptualizations of PPTEs that vary across studies, some focused on direct and indirect physical violence, whereas some included verbal aggression and threats, CWs consistently reported frequent exposures and relationships with PTSD $[12,13,15,46]$. In a study of Danish CWs, work-related threats increased the risk for PTSD, leading the authors to highlight the complexity involved in trying to capture the range of PPTEs experienced in the workplace and to speculate that, despite verbal threats appearing less immediately harmful than physical violence, the boundless nature of verbal threats may present a heavy psychological burden [46].

Perpetration of violence is typically attributed to prisoners, but few studies have examined the impact of collegial violence in correctional work [47,48]. Psychological harassment and intimidation of COs by their colleagues from other staff members has been associated with increased levels of distress [47], with the highest rates of PTSD among workers experiencing peer aggression [48]. Focusing on abuse perpetrated by prisoners' risks reinforcing stereotypes of prisoners as dangerous and risks missing important influences of $\mathrm{CW}$ mental health, particularly in relation to PPTEs and PTSD [49].

Interactions between occupational stressors and PPTE exposures have recently been associated with several mental health disorders (e.g., PTSD, MDD, GAD) among a sample of diverse Canadian PSP that included CWs [15]. Occupational stressors were significantly related to mental disorders even after controlling for exposure to PPTEs and CWs reported the highest organizational stress scores amongst all PSP occupations [15]. Including violence exposure and occupational stress variables in the same analysis allowed the researchers to conclude "that organizational and operational workplace stress might even play a larger role on PSP mental health than PPTEs" [15] (p. 19). The authors advocated for innovative solutions to occupational stressors as part of mitigating the significant mental health effects of PSP work.

\subsection{Correctional Worker Psychological Well-Being}

Researchers working with CW psychological well-being commonly focus their analyses on understanding risk factors contributing to job stress, burnout, and PTSD [12-14,22,50,51]. Some researchers conceptualize job stress as an outcome variable, while others analyze job 
stress as an antecedent to mental disorders [33,52,53]. For example, burnout can result from extended exposure to diverse stressors and may also be a risk factor for the development of PTSD [52]. Job stress is the most commonly measured psychological response within correctional research that explores the influence of various occupational stressors [3]; however, thresholds for identifying high levels of job stress remain inconsistent, and published prevalence rates for job stress amongst CWs are rare.

A recent systematic review was completed to estimate prevalence rates of mental health disorders (e.g., PTSD, MDD, GAD) amongst COs [6]. Only six articles met inclusion criteria for the review; specifically, having been published between 1980-2018, with data from COs, and providing prevalence measures of the mental disorder assessed. The resulting prevalence indicated COs were reporting more mental health challenges than the general population and "over three times the relevant national lifetime prevalence for PTSD" [6] (p. 8). In Canada, a nationwide survey of PSP estimated mental disorders prevalence amongst CWs in federal and provincial/territorial correctional systems at $54.6 \%$ [13], which was higher than other surveyed PSP groups, including police and firefighters. A follow-up study, focused on CWs in the Ontario provincial/territorial system, estimated mental disorders prevalence amongst CWs at $59 \%$, with women working as institutional COs screening positive for any mental disorder more frequently than men (see [14] for rates of PTSD, depression, and anxiety amongst Ontario CWs). Results from a study with Michigan Corrections Organization suggested over a third of COs working in high-security areas reported meeting criteria for PTSD or MDD, a quarter of their sample met the criteria for both, and 5 of 100 staff appeared at a high risk of death by suicide [11]. Overall, CWs today are more open about suffering from mental health challenges (e.g., PTSD) and lobbying for policy changes to improve access to mental health treatment across Canada $[54,55]$. Correspondingly, there is an increasing focus on shifting the outcome of the analysis from job stress to the mental health of CWs.

\subsection{Current Study}

The current study builds off the extensive body of scholarship on CO job stress and burnout by identifying the most prevalent stressors impacting the mental health of Ontario CWs. The inclusion of 43 occupational stressors in our study and measurement of specific mental disorders addresses some of the limitations in earlier research, which commonly focused its analysis on the significance of a single occupational stressor to employee job stress. Our study asks: what are the most salient stressors contributing to Ontario CW stress and mental health disorders? In order to answer this question, we first examined associations between overall scores for occupational stressors, as well as PPTEs, and positive screens for mental health disorders (e.g., PTSD, MDD). We expected significant positive associations among measures of stress (i.e., organizational and operational stressors) and screening positive for a mental health disorder. Second, we calculated the associations between organizational and operational stressors while controlling for PPTE exposure types. We expected the relationship between occupational stressors and positive screens for mental health disorders would remain statistically significant after controlling for exposure to PPTEs. We expected PPTE exposures and occupational stressors to be statistically significant moderators for screening positive for mental health disorders.

\section{Materials and Methods}

\subsection{Data and Sample}

The current study data were collected from the Ontario Correctional Worker Provincial Mental Health Prevalence Study collected via an Internet-delivered survey conducted from December 2017 to June 2018. Participants were employees within Ontario's Ministry of the Solicitor General who were working in correctional services (e.g., institutional, administrative, or community correctional services) and invited by email. The email invitations were sent by agency representatives from the Ontario Public Service Employees Union and the Ontario's Ministry of the Solicitor General and described the purpose of the 
study. The emails included an anonymous link that directed the employee to the survey introduction which provided more details about the study, informed consent, data storage procedures, data confidentiality, potential risks and resources, and study withdrawal processes. The invitation email could be forwarded and the overlap between the listservs used was unknown; therefore, we could not estimate the total number of people invited for potential participation or the participants' response rate. Further details on the study method are published elsewhere (removed for peer review).

A total of 1487 participants began the survey. Most participants $(n=1338)$ could be definitively placed into one of the six occupational categories under analysis: institutional wellness (e.g., psychologists, nurses, social workers), institutional training (e.g., program officers, teachers, volunteer coordinators), institutional governance (e.g., superintendents, assistant superintendents), COs, probation/parole officers, and institutional administration (e.g., payroll, administrative assistants). Participants whose occupational category was not consistent with one of the six categories of interest in this study were excluded from analyses. There were 907 participants $(67.8 \%$ of the 1338) who proceeded far enough in the survey to complete the modules required for the current study. There were 39 respondents who did not respond appropriately to an attention control question, resulting in a final analytic sample of $n=868(64.8 \%$ of the 1338 and $51.6 \%$ of the analytic sample selfidentified as female). There were no statistically significant differences in sociodemographic covariates or occupational categories noted between included or excluded participants from the current analyses (see supplementary online Table S1). Ethical approvals were obtained from the Queen's University and Affiliated Health Sciences Centre Research Ethics Board (file \#6024787), and the Research Ethics Boards at both the University of Regina (file \#2017-098), and Memorial University of Newfoundland (file \#20201330-EX).

\subsection{Measures}

\subsubsection{Occupational Stressors}

Occupational stressors were assessed with two standardized self-report questionnaires. The 20-item Organizational Police Stress Questionnaire (PSQ-Org) [56] assesses organizational stressors associated with performing a job (e.g., dealing with co-workers, staff shortages, excessive administrative duties) whereas the 20-item Operational Police Stress Questionnaire (PSQ-Op) assesses operational stressors related to the organization and culture within which the job is performed including the impact of work on family and social life (e.g., fatigue, shift work, making friends outside the job). The items in the scales are not specific to policing and the scale has been used in research on other public safety groups, including correctional officers [15]. Researchers developed an additional three stressors believed to be relevant to the correctional context which were also assessed (e.g., concern over job performance, communication across departments/branches, and working in close contact with the prisoner/probationer/parolee population). We provide a complete list of items in Table 1. Each item was assessed on a 7-point ordinal scale ranging from 1 (no stress at all) to 7 (a lot of stress). We computed scores for each individual PSQ-Org, PSQ-Op, and the three additional stressors items. Mean total scores on the PSQ-Org and PSQ-Op scales were computed by summing stress scores across all of the items and dividing by 20 . We computed mean total scores on the additional stressors scale by summing stress scores across all of the items and dividing by 3. Cronbach's alpha for the total mean scores were 0.93 for the PSQ-Org scale, 0.94 for the PSQ-Op scale, and 0.71 for the additional stressors scale, indicating that all of the measures were reliable measures of occupational stress in the study.

\subsubsection{Mental Disorder Symptoms}

The current study employed multiple reliable, validated self-report screening tools to assess for mental disorder symptoms. PTSD symptoms were assessed using the Life Events Checklist for the Diagnostic and Statistical Manual of Mental Disorders, 5th edition (LEC5) $[41,42]$ and the PCL-5 [42]. When responding to the PCL-5, in line with the Diagnostic 
and Statistical Manual of Mental Disorders, 5th edition (DSM-5) [44], participants were asked to report on their lifetime PPTE exposures, [41,42,57-59]. The LEC-5 does not include "sudden and unexpected death of someone close to you," as a PPTE, making the screening process arguably more conservative than some studies assessing positive screening frequencies with the PCL-5 [57]. When rating their past-month symptoms with the PCL-5, participants were asked to select an associated index PPTE (i.e., single worst PPTE, most distressing PPTE, or the PPTE currently causing the most distress). A participant screened positive on the PCL-5 if they met the minimum criteria for each PTSD cluster and exceeded the minimum clinical cutoff score of $>32$ in their total score [42]. MDD was assessed by having participants respond to the 9-item Patient Health Questionnaire (PHQ-9) and base their responses on the past 14-days [60-63]. A participant screened positive for MDD if their total score was $>9$ on the PHQ-9 [64]. GAD was assessed with the 7-item GAD scale (GAD-7) based on a past 14-day timeframe [60,62,65]. A participant screened positive for GAD if their total score was $>9$ on the GAD-7 [66]. Panic disorder was assessed with the 7-item Panic Disorder Symptoms Severity scale (PDSS) based on a past 7-day timeframe [67-69]. A participant screened positive for panic disorder if their total score was $>7$ on the PDSS scale [68]. Alcohol use disorder was assessed with the Alcohol Use Disorders Identification Test (AUDIT) based on a past 12-month timeframe [70,71]. A participant screened positive for alcohol use disorder if their total score was $>15$ on the AUDIT [70]. In lieu of screening tools, participants were asked to self-report whether they had been diagnosed with several other mental disorders (e.g., social anxiety disorder, obsessive-compulsive disorder, persistent depressive disorder, bipolar I, bipolar II, and cyclothymic disorder). The low prevalence of self-reported diagnosed disorders precluded the examination of each specific self-reported mental disorder with occupational stressors or PPTEs. As such, self-reported diagnosed disorders were only included in the dichotomous 'any positive mental disorder screen' variable, which was computed as positive if the participant screened positive on one or more of the screening tools and/or self-reported a mental disorder diagnosis.

\subsubsection{Total Number of PPTE Exposures}

The LEC-5 [41,42] assesses lifetime exposure to 16 PPTE (i.e., life-threatening natural disaster; fire or explosion; serious transportation accident; serious accident at work, home, or during recreational activity; exposure to a toxic substance; physical assault; assault with a weapon; sexual assault; other unwanted or uncomfortable sexual experience; combat; captivity; life-threatening illness or injury; severe human suffering; sudden violent death; sudden accidental death; serious injury, harm, or death you caused to someone else). For each participant, we summed exposures across the 16 items to arrive at the total number of PPTE exposures. We coded participant responses as having been exposed to a specific PPTE if they reported that: (a) it happened to them personally; (b) they witnessed it happen to someone else; (c) they learned about it happening to a close family member or close friend; and/or (d) they were exposed to it as part of their jobs as first responders. The percentage of missing responses on each individual item was small (ranged from $0.9 \%$ for physical assault to $10.1 \%$ for exposure to a toxic substance); nevertheless, cumulatively missing values compromised computation of the exact number of PPTE exposures for several participants. We allowed up to two missing values in the calculation of the total number of PPTE exposures variables (resulting in a further 146 respondents with 3 or more missing values being excluded from the study where the total number of traumatic exposures were considered). The mean number of PPTE exposures was $9.89(\mathrm{SD}=4.28)$ in the sample.

\subsubsection{Sociodemographic Covariates}

Sociodemographic covariates included sex (male or female), age (i.e., 19 to 29 years, 30 to 39 years, 40 to 49 years, 50 to 59 years, or 60 years and older), marital status (i.e., married/common-law, remarried, separated/divorced/widowed, or single), education 
(i.e., high school or less, some post-secondary less than 4-year college/university program, or university degree/4-year college or higher), total years of service (i.e., less than 4 years, 4 to 9 years, 10 to 15 years, or more than 15 years), and occupational category (i.e., institutional wellness, institutional training, institutional governance, correctional officers, probation/parole officers, and institutional administration). Covariates were chosen based on their association with mental disorder symptoms in previous studies (e.g., [13,14]).

\subsubsection{Statistical Analyses}

First, mean scores were computed for each individual occupational (i.e., organizational, operational, other) stressor and the total mean occupational scores (i.e., organizational, operational, other, total occupational stress) in the total sample. Across occupational categories of CWs, we identified differences in the mean levels of stress for all occupational stressors; and assessed for item-level differences in organizational (i.e., job context) and operational (i.e., job content) stressors within occupational categories. We expected variation across occupational categories, with COs, probation/parole officers, and institutional governance (e.g., superintendents) reporting the greatest levels of occupational stress, more so than institutional administrators (e.g., administrative assistants), wellness staff (e.g., nurses), and training staff (e.g., program officers). We tested differences across occupational categories using Bonferroni post hoc tests from a one-way ANOVA model. Second, multivariate logistic regression models were computed to examine the association between each individual occupational (i.e., organizational, operational, other) stressor and the total mean occupational scores (i.e., organizational, operational, other, and total occupational stress) and each type of positive mental disorder screen and any positive mental disorder screen. Logistic regression models adjusted for sociodemographic covariates (i.e., sex, age, marital status, education, province of residence, and total years of service), total number of PPTE exposures (range 0 to 16), and occupational category (i.e., institutional wellness, institutional training, institutional governance, correctional officer, probation/parole officer, or institutional administration). Third, we ran a series of nested multivariate logistic regression models to examine the independent and interactive effects of each type of mean occupational stress score (i.e., organizational, operational, and other stressors) and the total number of PPTE exposures on each type of positive mental disorder screen and any positive mental disorder screen. Logistic regression models adjusted for sociodemographic covariates and CW occupational category.

Missing data were minimal for sociodemographic covariates (ranged from $0 \%$ to $2.8 \%$ ) and the three separate occupational stressors scales (ranged from $0.2 \%$ to $1.5 \%$ ). Missing data on the mental disorder measures ranged from 0.2\% (MDD) to 8.0\% (any mental disorder). As stated previously, an additional 146 participants were excluded from analyses where total PPTE exposures were considered (due to three or more missing on the PPTE items). Missing data were excluded using complete case analyses in logistic regression models. Analyses were conducted using Stata (version 16.1) statistical software, Results at $p<0.05$ were considered statistically significant.

\section{Results}

Sociodemographic characteristics of the sample and the prevalence of positive mental disorder screens are provided in Table 1 . The sample was $48.4 \%$ male and $51.6 \%$ female (see Table 1). Most participants were married or living in a common-law relationship $(63.9 \%)$ and had completed at least some post-secondary education. Most participants were working as corrections officers $(58.1 \%)$ or as probation officers $(17.2 \%)$. The prevalence of positive mental disorder screens ranged from $6.8 \%$ (positive alcohol use disorder screen) to $37.1 \%$ (MDD) for each individual mental disorder. In total, $56.4 \%$ of the sample screened positive for one or more mental disorders. 
Table 1. Distribution of study variables in sample.

\begin{tabular}{|c|c|c|}
\hline Study Variable & $\%$ & $\mathbf{n}$ \\
\hline \multicolumn{3}{|l|}{ Sex } \\
\hline Male & 48.4 & 419 \\
\hline Female & 51.6 & 447 \\
\hline \multicolumn{3}{|l|}{ Age } \\
\hline 20 to 29 years & 18.3 & 158 \\
\hline 30 to 39 years & 29.0 & 250 \\
\hline 40 to 49 years & 26.7 & 230 \\
\hline 50 to 59 years & 23.9 & 206 \\
\hline 60 years and older & 2.1 & 18 \\
\hline \multicolumn{3}{|l|}{ Marital status } \\
\hline Married/common-law & 63.9 & 548 \\
\hline Single & 18.4 & 158 \\
\hline Separated/divorced/widowed & 14.0 & 120 \\
\hline Remarried & 3.6 & 31 \\
\hline \multicolumn{3}{|l|}{ Education } \\
\hline High school or less & 4.9 & 41 \\
\hline $\begin{array}{l}\text { Some post-secondary (less than 4-year } \\
\text { college/university degree) }\end{array}$ & 46.6 & 393 \\
\hline 4-year college program/university degree & 48.5 & 409 \\
\hline \multicolumn{3}{|l|}{ Years of Service } \\
\hline Less than 4 years & 29.8 & 256 \\
\hline 4 to 9 years & 12.5 & 107 \\
\hline 10 to 15 years & 18.6 & 160 \\
\hline More than 15 years & 39.1 & 336 \\
\hline \multicolumn{3}{|l|}{ Occupational Category } \\
\hline Wellness & 8.4 & 73 \\
\hline Training & 3.7 & 32 \\
\hline Governance & 9.5 & 82 \\
\hline Correctional Officers & 58.1 & 504 \\
\hline Probation/Parole Officers & 17.2 & 149 \\
\hline Administration & 3.2 & 28 \\
\hline \multicolumn{3}{|l|}{ Positive PTSD Screen } \\
\hline No & 70.3 & 582 \\
\hline Yes & 29.7 & 246 \\
\hline \multicolumn{3}{|l|}{ Positive Depression Screen } \\
\hline No & 62.9 & 545 \\
\hline Yes & 37.1 & 321 \\
\hline \multicolumn{3}{|l|}{ Positive Generalized Anxiety Screen } \\
\hline No & 68.8 & 593 \\
\hline Yes & 31.2 & 269 \\
\hline
\end{tabular}


Table 1. Cont.

\begin{tabular}{ccc}
\hline Study Variable & \% & $\mathbf{n}$ \\
\hline Positive Panic Disorder Screen & & 685 \\
\hline No & 85.7 & 114 \\
\hline Yes & 14.3 & \\
\hline Positive Alcohol Use Disorder Screen & 788 \\
\hline No & 93.3 & 57 \\
\hline Yes & 6.8 & \\
\hline Any Positive Mental Disorder Screen & & 348 \\
\hline No & 43.6 & 450 \\
\hline Yes & 56.4 & \\
\hline
\end{tabular}

We provide the mean stress levels associated with occupational stressors (organizational, operational, and other stressors) across CW occupational categories in Table 2. In the total sample, the organizational stressors associated with the highest mean levels of stress were staff shortages (4.89), inconsistent leadership style (4.76), bureaucratic red tape (4.66), feeling that different rules apply to different people (4.61), lack of resources (4.52), constant changes in policy and legislation (4.45), and dealing with co-workers (4.40). Organizational stressors were also reported as those with the highest mean level of stress across $\mathrm{CW}$ occupational categories; however, statistically significant differences in the actual mean scores existed across occupational categories. Correctional employees working in institutional wellness, training, or administration tended to report lower mean stress scores across organizational stressors than those working in institutional governance, COs, and probation/parole officers. There were organizational stressors that appeared particularly salient for employees working as probation/parole officers; specifically, probation/parole officers tended to report higher mean levels of stress associated with excessive administrative duties (5.07), too much computer work (4.99), and dealing with the court system (3.69) than other occupational categories.

The mean level of stress associated with operational stressor items (total mean score 3.31) tended to be lower than the mean level of stress associated with organizational stressor items (total mean score 3.87). In the total sample, the operational stressors associated with the highest mean levels of stress were finding time to stay in good physical condition (4.39), fatigue (4.24), occupation-related health issues (3.95), not enough time available to spend with friends and family (3.93), eating healthy at work (3.78), risk of being injured on the job (3.73), and lack of understanding from family and friends about your work (3.72). Again, the operational stressors associated with the highest level of stress were similar across $\mathrm{CW}$ occupational categories, although significant differences in the actual mean scores existed across occupational categories. Employees working in institutional governance and COs tended to report the highest mean stress scores across organizational stressors. Moreover, there were a few operational stressors that seemed associated with higher levels of stress in specific CW occupational groups. Probation/parole officers tended to report higher mean levels of stress associated with paperwork (5.03), employees working in institutional governance tended to report higher mean levels of stress associated with over-time demands (3.44), and COs tended to report higher mean levels of stress associated with fatigue (4.82) and the risk of being injured on the job (4.56) than other occupational categories. 
Table 2. Mean stress levels associated with occupational stressors across correction worker occupational categories in Ontario, Canada.

\begin{tabular}{|c|c|c|c|c|c|c|c|c|c|}
\hline \multirow[t]{2}{*}{ Occupational Stressor } & Total & Wellness $^{a}$ & Training ${ }^{b}$ & Governance $^{c}$ & $\begin{array}{l}\text { Correctional } \\
\text { Officers }^{\mathrm{d}}\end{array}$ & $\begin{array}{l}\text { Probation/Parole } \\
\text { Officers e }\end{array}$ & Administration ${ }^{\mathrm{f}}$ & \multirow[t]{2}{*}{ F-Statistic } & \multirow{2}{*}{$\begin{array}{l}\text { Significant Differences } \\
\text { between Occupational } \\
\text { Categories }\end{array}$} \\
\hline & Mean (SE) & Mean (SD) & Mean (SD) & Mean (SD) & Mean (SD) & Mean (SD) & Mean (SD) & & \\
\hline \multicolumn{10}{|l|}{ Organizational Stressors (PSP-Org) } \\
\hline Dealing with co-workers & $4.40(0.06)$ & $4.63(0.20)$ & $4.34(0.35)$ & $4.94(0.17)$ & $4.36(0.08)$ & $4.17(0.15)$ & $4.39(0.43)$ & $2.29 *$ & $\mathrm{e}<\mathrm{c}$ \\
\hline $\begin{array}{l}\text { The feeling that different rules apply to } \\
\text { different people (e.g., favouritism) }\end{array}$ & $4.61(0.06)$ & $4.75(0.24)$ & $4.38(0.36)$ & $4.85(0.20)$ & $4.68(0.08)$ & $4.20(0.16)$ & $4.68(0.43)$ & 2.00 & No significant differences \\
\hline $\begin{array}{l}\text { Feeling like you always have to prove } \\
\text { yourself to the organization }\end{array}$ & $4.08(0.07)$ & $3.66(0.23)$ & $3.88(0.36)$ & $4.55(0.23)$ & $4.04(0.09)$ & $4.20(0.16)$ & $1.04(0.41)$ & 1.81 & No significant differences \\
\hline Excessive administrative duties & $3.48(0.07)$ & $3.66(0.23)$ & $3.47(0.36)$ & $4.45(0.21)$ & $2.83(0.08)$ & $5.07(0.15)$ & $3.54(0.44)$ & $40.82 * * *$ & $\begin{array}{c}a<d \\
a, b, d, f<e\end{array}$ \\
\hline Constant change in policy/legislation & $4.45(0.07)$ & $3.59(0.21)$ & $3.25(0.33)$ & $4.78(0.20)$ & $4.54(0.08)$ & $4.81(0.15)$ & $3.57(0.35)$ & $8.69^{* * *}$ & $\begin{array}{c}\mathrm{a}, \mathrm{b}<\mathrm{c}, \mathrm{d}, \mathrm{e} \\
\mathrm{f}<\mathrm{e}\end{array}$ \\
\hline Staff shortages & $4.89(0.07)$ & $5.00(0.24)$ & $4.13(0.39)$ & $5.20(0.20)$ & $4.79(0.09)$ & $5.20(0.15)$ & $4.61(0.38)$ & $2.54^{*}$ & No significant differences \\
\hline Bureaucratic red tape & $4.66(0.07)$ & $4.14(0.26)$ & $3.81(0.40)$ & $5.06(0.18)$ & $4.66(0.09)$ & $4.99(0.15)$ & $4.25(0.38)$ & $3.84 * *$ & $\begin{array}{c}\mathrm{a}<\mathrm{e} \\
\mathrm{b}<\mathrm{c}, \mathrm{e}\end{array}$ \\
\hline Too much computer work & $3.00(0.07)$ & $2.81(0.21)$ & $3.00(0.32)$ & $3.94(0.21)$ & $2.28(0.07)$ & $4.99(0.16)$ & $3.04(0.41)$ & $65.79 * * *$ & $\begin{array}{c}\mathrm{a}, \mathrm{b}, \mathrm{c}, \mathrm{d}, \mathrm{f}<\mathrm{e} \\
\mathrm{a}, \mathrm{d}<\mathrm{c}\end{array}$ \\
\hline Perceived pressure to volunteer free time & $2.12(0.06)$ & $1.85(0.18)$ & $1.97(0.32)$ & $2.52(0.21)$ & $2.05(0.07)$ & $2.27(0.14)$ & $2.32(0.36)$ & 1.99 & No significant differences \\
\hline Dealing with supervisors & $4.06(0.07)$ & $3.45(0.23)$ & $3.13(0.37)$ & $4.16(0.22)$ & $4.29(0.09)$ & $3.78(0.15)$ & $3.89(0.43)$ & $5.03 * * *$ & $\mathrm{a}, \mathrm{b}<\mathrm{d}$ \\
\hline Inconsistent leadership style & $4.76(0.07)$ & $4.13(0.26)$ & $4.09(0.40)$ & $4.68(0.22)$ & $5.14(0.08)$ & $4.04(0.16)$ & $4.21(0.48)$ & $10.48^{* * *}$ & $\mathrm{a}, \mathrm{e}<\mathrm{d}$ \\
\hline Lack of resources & $4.52(0.07)$ & $4.36(0.25)$ & $4.16(0.35)$ & $4.48(0.22)$ & $4.64(0.09)$ & $4.41(0.17)$ & $3.79(0.41)$ & 1.56 & No significant differences \\
\hline Unequal sharing of work responsibilities & $4.26(0.07)$ & $4.49(0.25)$ & $3.81(0.43)$ & $4.23(0.21)$ & $4.36(0.09)$ & $3.99(0.17)$ & $3.93(0.49)$ & 1.39 & No significant differences \\
\hline $\begin{array}{l}\text { If you are sick or injured your co-workers } \\
\text { seem to look down on you }\end{array}$ & $3.09(0.07)$ & $2.71(0.25)$ & $2.13(0.29)$ & $3.23(0.24)$ & $3.29(0.10)$ & $2.71(0.15)$ & $2.93(0.46)$ & $3.65^{* *}$ & $\mathrm{~b}<\mathrm{d}$ \\
\hline $\begin{array}{l}\text { Leaders over-emphasize the negatives } \\
\text { (e.g., supervisor evaluations, public } \\
\text { complaints) }\end{array}$ & $3.95(0.07)$ & $3.01(0.24)$ & $2.63(0.38)$ & $4.16(0.23)$ & $4.18(0.10)$ & $3.85(0.18)$ & $3.54(0.51)$ & $6.67^{* * *}$ & $\mathrm{a}, \mathrm{b}<\mathrm{c}, \mathrm{d}$ \\
\hline Internal investigations & $3.52(0.08)$ & $3.14(0.28)$ & $2.50(0.34)$ & $3.77(0.23)$ & $3.90(0.11)$ & $2.74(0.17)$ & $2.25(0.34)$ & $9.94 * * *$ & $\begin{array}{c}\mathrm{b}, \mathrm{e}, \mathrm{f}<\mathrm{d} \\
\mathrm{e}, \mathrm{f}<\mathrm{c}\end{array}$ \\
\hline Dealing with the court system & $2.56(0.06)$ & $2.34(0.19)$ & $2.09(0.30)$ & $2.61(0.18)$ & $2.27(0.07)$ & $3.69(0.14)$ & $2.64(0.39)$ & $17.75^{* * *}$ & $\mathrm{a}, \mathrm{b}, \mathrm{c}, \mathrm{d}, \mathrm{f}<\mathrm{e}$ \\
\hline $\begin{array}{l}\text { The need to be accountable for doing } \\
\text { your job }\end{array}$ & $3.91(0.07)$ & $3.37(0.24)$ & $3.03(0.37)$ & $4.05(0.20)$ & $3.85(0.09)$ & $4.51(0.16)$ & $3.71(0.47)$ & $5.18^{* * *}$ & $\mathrm{a}, \mathrm{b}, \mathrm{d}<\mathrm{e}$ \\
\hline
\end{tabular}


Table 2. Cont

\begin{tabular}{|c|c|c|c|c|c|c|c|c|c|}
\hline \multirow[t]{2}{*}{ Occupational Stressor } & Total & Wellness $^{a}$ & Training ${ }^{b}$ & Governance $^{c}$ & $\begin{array}{c}\text { Correctional } \\
\text { Officers }^{d}\end{array}$ & $\begin{array}{c}\text { Probation/Parole } \\
\text { Officers e }\end{array}$ & Administration ${ }^{\mathrm{f}}$ & \multirow[t]{2}{*}{ F-Statistic } & \multirow{2}{*}{$\begin{array}{c}\text { Significant Differences } \\
\text { between Occupational } \\
\text { Categories }\end{array}$} \\
\hline & Mean (SE) & Mean (SD) & Mean (SD) & Mean (SD) & Mean (SD) & Mean (SD) & & & \\
\hline Inadequate equipment & $3.65(0.07)$ & $3.51(0.26)$ & $2.97(0.37)$ & $3.61(0.24)$ & $3.96(0.09)$ & $3.04(0.17)$ & $2.50(0.34)$ & $7.75^{* * *}$ & $\mathrm{e}, \mathrm{f}<\mathrm{d}$ \\
\hline $\begin{array}{c}\text { Total Mean Organizational Stress Score, } \\
\text { mean (SD) }\end{array}$ & $3.87(0.04)$ & $3.59(0.14)$ & $3.28(0.23)$ & $4.14(0.15)$ & $3.89(0.06)$ & $3.99(0.10)$ & $3.54(0.31)$ & $3.34 * *$ & $\mathrm{~b}<\mathrm{c}$ \\
\hline \multicolumn{10}{|l|}{ Operational Stressors (PSP-Op) } \\
\hline Shift work & $3.36(0.08)$ & $2.49(0.26)$ & $1.34(0.20)$ & $3.29(0.25)$ & $4.42(0.09)$ & $1.09(0.05)$ & $1.21(0.15)$ & $93.96^{* * *}$ & $\begin{array}{c}\mathrm{a}<\mathrm{d}, \mathrm{e}, \mathrm{f} \\
\quad \mathrm{c}<\mathrm{d} \\
\mathrm{b}, \mathrm{e}, \mathrm{f}<\mathrm{c}, \mathrm{d}\end{array}$ \\
\hline Working alone at night & $2.23(0.07)$ & $2.14(0.26)$ & $1.00(0.0)$ & $2.23(0.22)$ & $2.68(0.09)$ & $1.24(0.07)$ & $1.00(0.00)$ & $20.26^{* * *}$ & $\begin{array}{c}\mathrm{b}, \mathrm{e}<\mathrm{a}, \mathrm{c}, \mathrm{d} \\
\mathrm{f}<\mathrm{c}, \mathrm{d}\end{array}$ \\
\hline Over-time demands & $2.51(0.06)$ & $2.23(0.21)$ & $1.19(0.11)$ & $3.44(0.25)$ & $2.66(0.08)$ & $2.05(0.14)$ & $1.68(0.24)$ & $11.30 * * *$ & $\begin{array}{c}\mathrm{a}, \mathrm{b}, \mathrm{d}, \mathrm{e}, \mathrm{f}<\mathrm{c} \\
\mathrm{b}<\mathrm{c}, \mathrm{d} \\
\mathrm{e}<\mathrm{d}\end{array}$ \\
\hline Risk of being injured on the job & $3.73(0.07)$ & $2.64(0.19)$ & $2.53(0.33)$ & $3.20(0.21)$ & $4.56(0.08)$ & $2.40(0.13)$ & $1.75(0.26)$ & 54.50 & $\begin{array}{c}\mathrm{a}, \mathrm{b}, \mathrm{c}, \mathrm{e}, \mathrm{f}<\mathrm{d} \\
\mathrm{c}<\mathrm{e}, \mathrm{f}\end{array}$ \\
\hline $\begin{array}{l}\text { Work-related activities on days off (e.g., } \\
\text { court, community events) }\end{array}$ & $2.00(0.05)$ & $1.53(0.13)$ & $1.34(0.16)$ & $2.09(0.17)$ & $2.11(0.07)$ & $2.04(0.13)$ & $1.57(0.25)$ & $3.67^{* *}$ & $\mathrm{a}<\mathrm{d}$ \\
\hline $\begin{array}{l}\text { Traumatic events (e.g., motor vehicle } \\
\text { accidents, domestics, death, injury) }\end{array}$ & $2.82(0.07)$ & $2.21(0.21)$ & $1.94(0.24)$ & $2.87(0.20)$ & $3.02(0.09)$ & $2.72(0.15)$ & $2.07(0.34)$ & $4.79^{* * *}$ & $\mathrm{a}, \mathrm{b}<\mathrm{d}$ \\
\hline Managing your social life outside of work & $3.28(0.06)$ & $2.58(0.19)$ & $2.50(0.31)$ & $3.29(0.21)$ & $3.62(0.08)$ & $2.77(0.14)$ & $2.64(0.34)$ & $9.67^{* * *}$ & $\mathrm{a}, \mathrm{b}, \mathrm{e}<\mathrm{d}$ \\
\hline $\begin{array}{l}\text { Not enough time available to spend with } \\
\text { friends and family }\end{array}$ & $3.93(0.07)$ & $3.04(0.25)$ & $3.47(0.41)$ & $4.01(0.24)$ & $4.39(0.09)$ & $3.03(0.16)$ & $3.00(0.39)$ & $15.64^{* * *}$ & $\begin{array}{c}\mathrm{a}, \mathrm{e}<\mathrm{c}, \mathrm{d} \\
\mathrm{f}<\mathrm{d}\end{array}$ \\
\hline Paperwork & $3.41(0.07)$ & $3.51(0.25)$ & $2.78(0.33)$ & $4.09(0.20)$ & $2.86(0.08)$ & $5.03(0.16)$ & $3.07(0.39)$ & $35.58^{* * *}$ & $\begin{array}{c}\mathrm{a}, \mathrm{b}, \mathrm{d}, \mathrm{d}, \mathrm{f}<\mathrm{e} \\
\mathrm{b}, \mathrm{d}<\mathrm{c}\end{array}$ \\
\hline Eating healthy at work & $3.78(0.07)$ & $3.21(0.22)$ & $3.53(0.37)$ & $3.94(0.21)$ & $3.97(0.09)$ & $3.48(0.16)$ & $3.39(0.40)$ & $3.40 * *$ & $\mathrm{a}<\mathrm{d}$ \\
\hline $\begin{array}{l}\text { Finding time to stay in good physical } \\
\text { condition }\end{array}$ & $4.39(0.07)$ & $3.90(0.23)$ & $4.13(0.39)$ & $4.66(0.20)$ & $4.49(0.08)$ & $4.23(0.16)$ & $4.14(0.36)$ & 1.96 & No significant differences \\
\hline Fatigue (e.g., shift work, over-time) & $4.24(0.07)$ & $3.44(0.23)$ & $2.47(0.34)$ & $4.34(0.24)$ & $4.82(0.09)$ & $3.27(0.18)$ & $2.79(0.42)$ & $25.76^{* * *}$ & $\begin{array}{c}\mathrm{a}<\mathrm{d} \\
\mathrm{b}, \mathrm{e}, \mathrm{f}<\mathrm{c}, \mathrm{d}\end{array}$ \\
\hline $\begin{array}{l}\text { Occupation-related health issues (e.g., } \\
\text { back pain) }\end{array}$ & $3.95(0.07)$ & $3.67(0.27)$ & $2.78(0.33)$ & $3.93(0.23)$ & $4.13(0.09)$ & $3.83(0.17)$ & $3.36(0.39)$ & $3.55^{* *}$ & $\mathrm{~b}<\mathrm{d}$ \\
\hline
\end{tabular}


Table 2. Cont.

\begin{tabular}{|c|c|c|c|c|c|c|c|c|c|}
\hline \multirow[t]{2}{*}{ Occupational Stressor } & Total & Wellness $^{\text {a }}$ & Training ${ }^{b}$ & Governance $^{c}$ & $\begin{array}{c}\text { Correctional } \\
\text { Officers }{ }^{d}\end{array}$ & $\begin{array}{c}\text { Probation/Parole } \\
\text { Officers e }\end{array}$ & Administration $\mathrm{f}$ & \multirow[t]{2}{*}{ F-Statistic } & \multirow{2}{*}{$\begin{array}{c}\text { Significant Differences } \\
\text { between Occupational } \\
\text { Categories }\end{array}$} \\
\hline & Mean (SE) & Mean (SD) & Mean (SD) & Mean (SD) & Mean (SD) & Mean (SD) & Mean (SD) & & \\
\hline $\begin{array}{l}\text { Lack of understanding from family and } \\
\text { friends about your work }\end{array}$ & $3.72(0.07)$ & $2.55(0.22)$ & $2.75(0.37)$ & $3.55(0.23)$ & $4.12(0.09)$ & $3.46(0.16)$ & $2.64(0.38)$ & $12.81^{* * *}$ & $\begin{array}{c}a<c, e \\
a, b, e, f<d\end{array}$ \\
\hline Making friends outside the job & $3.01(0.07)$ & $2.21(0.21)$ & $2.38(0.34)$ & $2.93(0.22)$ & $3.37(0.09)$ & $2.46(0.15)$ & $2.75(0.39)$ & $8.35^{* * *}$ & $\mathrm{a}, \mathrm{e}<\mathrm{d}$ \\
\hline Upholding a "higher image" in public & $2.92(0.07)$ & $1.97(0.18)$ & $2.28(0.31)$ & $2.86(0.20)$ & $3.20(0.09)$ & $2.72(0.16)$ & $2.50(0.39)$ & $6.54^{* * *}$ & $\mathrm{a}<\mathrm{d}$ \\
\hline Negative comments from the public & $3.43(0.07)$ & $2.21(0.19)$ & $2.47(0.34)$ & $3.33(0.22)$ & $3.87(0.10)$ & $3.01(0.16)$ & $2.18(0.36)$ & $14.37^{* * *}$ & $\begin{array}{l}\mathrm{a}, \mathrm{b}<\mathrm{c} \\
\mathrm{a}, \mathrm{e}, \mathrm{f}<\mathrm{d}\end{array}$ \\
\hline $\begin{array}{l}\text { Limitations to your social life (e.g., who } \\
\text { your friends are, where you socialize) }\end{array}$ & $3.21(0.07)$ & $2.07(0.18)$ & $2.31(0.31)$ & $2.96(0.20)$ & $3.57(0.09)$ & $2.96(0.16)$ & $2.86(0.41)$ & $10.48^{* * *}$ & $\begin{array}{c}\mathrm{a}<\mathrm{e} \\
\mathrm{a}, \mathrm{b}, \mathrm{e}<\mathrm{d}\end{array}$ \\
\hline Feeling like you are always on the job & $3.33(0.07)$ & $2.14(0.20)$ & $2.22(0.31)$ & $3.55(0.22)$ & $3.63(0.09)$ & $3.21(0.17)$ & $2.25(0.35)$ & $11.17^{* * *}$ & $\begin{array}{c}\mathrm{a}<\mathrm{c}, \mathrm{e} \\
\mathrm{b}<\mathrm{c} \\
\mathrm{a}, \mathrm{b}, \mathrm{e}, \mathrm{f}<\mathrm{d}\end{array}$ \\
\hline $\begin{array}{l}\text { Friends / family feel the effects of the } \\
\text { stigma associated with your job }\end{array}$ & $3.10(0.07)$ & $1.77(0.15)$ & $2.56(0.35)$ & $3.24(0.20)$ & $3.50(0.09)$ & $2.61(0.15)$ & $2.36(0.38)$ & $14.82 * * *$ & $\begin{array}{l}\mathrm{a}<\mathrm{c}, \mathrm{d}, \mathrm{e} \\
\mathrm{e}, \mathrm{f}<\mathrm{d}\end{array}$ \\
\hline $\begin{array}{l}\text { Total Mean Operational Stress Score, } \\
\text { mean (SD) }\end{array}$ & $3.31(0.05)$ & $2.60(0.13)$ & $2.40(0.19)$ & $3.37(0.15)$ & $3.64(0.06)$ & $2.89(0.10)$ & $2.46(0.23)$ & $19.17^{* * *}$ & $\begin{array}{l}a, b, f<c \\
a, b, e, f<d\end{array}$ \\
\hline Concern over job performance & $3.55(0.07)$ & $2.88(0.20)$ & $3.09(0.39)$ & $3.79(0.22)$ & $3.52(0.09)$ & $4.12(0.16)$ & $2.71(0.42)$ & $5.88^{* * *}$ & $\mathrm{a}, \mathrm{d}, \mathrm{f}<\mathrm{e}$ \\
\hline $\begin{array}{l}\text { Communication across departments/ } \\
\text { branches }\end{array}$ & $3.62(0.07)$ & $3.30(0.23)$ & $3.34(0.40)$ & $4.09(0.1)$ & $3.69(0.09)$ & $3.42(0.16)$ & $3.32(0.42)$ & 1.82 & No significant differences \\
\hline $\begin{array}{l}\text { Working in close contact with the } \\
\text { inmate/client population }\end{array}$ & $3.43(0.07)$ & $2.75(0.21)$ & $2.47(0.29)$ & $2.83(0.21)$ & $3.78(0.09)$ & $3.40(0.15)$ & $1.86(0.29)$ & $11.36^{* * *}$ & $\begin{array}{c}\mathrm{a}, \mathrm{b}, \mathrm{c}, \mathrm{f}<\mathrm{d} \\
\mathrm{f}<\mathrm{e}\end{array}$ \\
\hline $\begin{array}{c}\text { Total Mean Other Stressors Score, } \\
\text { mean (SD) }\end{array}$ & $3.53(0.05)$ & $2.98(0.15)$ & $2.97(0.31)$ & $3.57(0.16)$ & $3.66(0.07)$ & $3.65(0.12)$ & $2.63(0.33)$ & $5.17^{* * *}$ & $\begin{array}{c}\mathrm{a}, \mathrm{f}<\mathrm{d} \\
\mathrm{f}<\mathrm{e}\end{array}$ \\
\hline
\end{tabular}

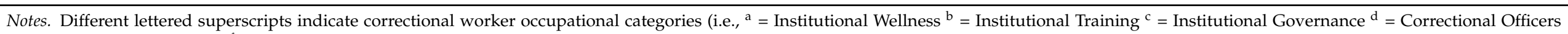

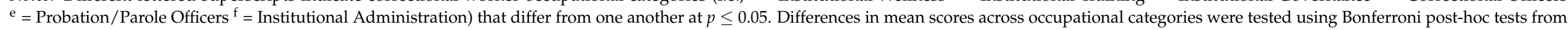
the one-way ANOVA models. ${ }^{*} p \leq 0.05 ;{ }^{* *} p \leq 0.01{ }^{* * *} p \leq 0.001$. 
Mean levels of stress associated with other stressors in the total sample were working in close contact with the prison/probationer/parolee population (3.43), concern over job performance (3.55), and communication across departments/branches (3.62). Probation/parole officers reported higher mean levels of stress related to concerns over job performance (4.12) than institutional wellness, training, or administrative employees. COs reported higher mean levels of stress concerning working in close contact with the prison population (3.78) than all other occupational groups except probation/parole officers.

Associations between occupational stressors (i.e., organizational, operational, other stressors) and positive mental disorder screens are presented in Table 3. All the individual types of organizational, operational, and other stressors were associated with increased odds of positive screens for PTSD (adjusted odds ratios [AOR] ranged from 1.15 to 1.52), MDD (AORs ranged from 1.21 to 1.86), GAD (AORs ranged from 1.22 to 2.00), panic disorder (AORs ranged from 1.18 to 1.76), and any mental disorder (AORs ranged from 1.25 to 1.80) after adjustment for sociodemographic covariates, total number of PPTE exposures, and occupational category. For alcohol abuse, 5 of 20 organizational stressors (i.e., dealing with co-workers, constant change in policy/legislation, too much computer work, unequal sharing of work responsibilities, and if you are sick or injured your co-workers seem to look down on you), 17 of 20 operational stressors (significant AORs ranged from 1.17 to 1.51), and 1 of 3 additional stressors (i.e., communication across departments/branches) were associated with increased odds of a positive alcohol abuse screen after adjustment for sociodemographic covariates and the total number of PPTE exposures.

The independent and interactive effects of mean occupational stress scores (i.e., mean scores on all three separate occupational stress subscales) and total number of PPTE exposures (range from 0 to 16) by type of positive mental disorder screen are provided in Table 4. Except for the relationship between the total number of PPTE exposures and alcohol use disorders, both the total number of PPTE exposures (AORs ranged from 1.07 to 1.17) and the mean operational stress scores (AORs ranged from 1.77 to 2.43) were associated with each individual mental disorder and any mental disorder when entered into the models independently (i.e., models 1 and 2), after adjusting for sociodemographic covariates. Organizational stressors were associated with PTSD, depression, and any positive mental disorders (AORs ranged from 1.40 to 1.49) in models adjusting for sociodemographic covariates and other types of occupational stressors. The other stressors subscale was not significantly associated with positive mental disorder screens after adjustment for sociodemographic covariates and other types of occupational stressors (i.e., model 2). For alcohol use, the mean occupational stress score ( $\mathrm{AOR}=1.77,95 \% \mathrm{CI}=1.22,2.58)$, but not total number of PPTEs, was independently associated with a positive alcohol use disorder screen. When the total number of PPTE exposures and mean occupational stress scores were entered into logistic regression models simultaneously (i.e., model 3), total number of PPTE exposures ( $\mathrm{AOR}=1.11,95 \% \mathrm{CI}=1.04,1.17)$, mean organizational stress score $(\mathrm{AOR}=1.34,95 \% \mathrm{CI}=1.03,1.76)$, and mean operational stress scores $(\mathrm{AOR}=1.74$, $95 \% \mathrm{CI}=1.34,2.26$ ) remained independently associated with increased odds of positive screens for PTSD after adjustment for sociodemographic covariates. In model 3, the total number of PPTE exposures was no longer statistically significantly associated with MDD, GAD, panic disorder, or any positive mental disorder screen when entered into the model simultaneously with mean occupational stress scores across all of the assessed mental disorders. In model 3, the mean organizational stress score also remained significantly associated with $\mathrm{MDD}(\mathrm{AOR}=1.64,95 \% \mathrm{CI}=1.26,2.12)$ and any positive mental disorder screen $(\mathrm{AOR}=1.55,95 \% \mathrm{CI}=1.20,2.00)$ and mean operational stress scores remained significantly associated with all other mental disorders and any mental disorder (AORs ranged from 1.93 to 2.37) after adjustment for sociodemographic covariates, total number of PPTE exposures, and other types of occupational stressors. A significant interaction was detected for total number of PPTE exposures by mean organizational stress interaction on MDD (see Figure 1). None of the other total number of PPTE exposures by mean occupational stress score interaction terms were statistically significant (i.e., model 4). 
Table 3. Relationship between occupational stressors and positive mental disorder screens among correction workers in Ontario, Canada.

\begin{tabular}{|c|c|c|c|c|c|c|}
\hline \multirow{2}{*}{ Occupational Stressor } & PTSD $^{a}$ & $\begin{array}{l}\text { Major Depressive } \\
\text { Disorder }\end{array}$ & $\begin{array}{l}\text { Generalized } \\
\text { Anxiety }\end{array}$ & Panic Disorder & Alcohol Use Disorder ${ }^{b}$ & Any Mental Disorder \\
\hline & $\begin{array}{c}\text { AOR } \\
(95 \% \mathrm{CI})\end{array}$ & $\begin{array}{c}\text { AOR } \\
(95 \% \mathrm{CI})\end{array}$ & $\begin{array}{c}\text { AOR } \\
(95 \% \mathrm{CI})\end{array}$ & $\begin{array}{c}\text { AOR } \\
(95 \% \mathrm{CI})\end{array}$ & $\begin{array}{c}\text { AOR } \\
(95 \% \mathrm{CI})\end{array}$ & $\begin{array}{c}\text { AOR } \\
(95 \% \mathrm{CI})\end{array}$ \\
\hline Dealing with co-workers & $\begin{array}{c}1.37^{* * *} \\
(1.22,1.54)\end{array}$ & $\begin{array}{c}1.58^{* * * *} \\
(1.41,1.77)\end{array}$ & $\begin{array}{c}1.55^{* * *} \\
(1.38,1.74)\end{array}$ & $\begin{array}{c}1.51^{* * *} \\
(1.29,1.77)\end{array}$ & $\begin{array}{c}1.27^{*} \\
(1.04,1.56)\end{array}$ & $\begin{array}{c}1.48^{* * *} \\
(1.33,1.65)\end{array}$ \\
\hline $\begin{array}{l}\text { The feeling that different rules apply to different } \\
\text { people (e.g., favouritism) }\end{array}$ & $\begin{array}{c}1.37^{* * *} \\
(1.22,1.53) \\
\end{array}$ & $\begin{array}{c}1.53^{* * *} \\
(1.38,1.71) \\
\end{array}$ & $\begin{array}{c}1.47^{* * *} \\
(1.31,1.64) \\
\end{array}$ & $\begin{array}{c}1.49^{* * *} \\
(1.26,1.75) \\
\end{array}$ & $\begin{array}{c}1.21 \\
(0.997,1.48) \\
\end{array}$ & $\begin{array}{c}1.44^{* * *} \\
(1.30,1.59) \\
\end{array}$ \\
\hline $\begin{array}{l}\text { Feeling like you always have to prove yourself to } \\
\text { the organization }\end{array}$ & $\begin{array}{c}1.49^{* * *} \\
(1.25,1.54)\end{array}$ & $\begin{array}{c}1.42^{* * *} \\
(1.29,1.57)\end{array}$ & $\begin{array}{c}1.46^{* * *} \\
(1.32,1.62)\end{array}$ & $\begin{array}{c}1.48^{* * *} \\
(1.29,1.71)\end{array}$ & $\begin{array}{c}1.12 \\
(0.94,1.33)\end{array}$ & $\begin{array}{c}1.48^{* * *} \\
(1.34,1.64)\end{array}$ \\
\hline Excessive administrative duties & $\begin{array}{c}1.30^{* * *} \\
(1.17,1.45)\end{array}$ & $\begin{array}{c}1.48^{* * *} \\
(1.33,1.64)\end{array}$ & $\begin{array}{c}1.40^{* * * *} \\
(1.26,1.56)\end{array}$ & $\begin{array}{c}1.51^{* * *} \\
(1.31,1.75)\end{array}$ & $\begin{array}{c}1.17 \\
(0.99,1.39)\end{array}$ & $\begin{array}{c}1.48^{* * *} \\
(1.33,1.65)\end{array}$ \\
\hline Constant change in policy/legislation & $\begin{array}{c}1.41^{* * *} \\
(1.26,1.58)\end{array}$ & $\begin{array}{c}1.50^{* * *} \\
(1.35,1.67)\end{array}$ & $\begin{array}{c}1.53 * * * \\
(1.37,1.71)\end{array}$ & $\begin{array}{c}1.49^{* * *} \\
(1.28,1.75)\end{array}$ & $\begin{array}{c}1.28^{*} \\
(1.06,1.56)\end{array}$ & $\begin{array}{c}1.55^{* * *} \\
(1.40,1.72)\end{array}$ \\
\hline Staff shortages & $\begin{array}{c}1.33^{* * *} \\
(1.19,1.50) \\
\end{array}$ & $\begin{array}{c}1.38^{* * *} \\
(1.24,1.54) \\
\end{array}$ & $\begin{array}{c}1.35^{* * *} \\
(1.21,1.50) \\
\end{array}$ & $\begin{array}{c}1.34^{* * *} \\
(1.14,1.57) \\
\end{array}$ & $\begin{array}{c}1.14 \\
(0.94,1.39) \\
\end{array}$ & $\begin{array}{c}1.39^{* * *} \\
(1.26,1.54) \\
\end{array}$ \\
\hline Bureaucratic red tape & $\begin{array}{c}1.37^{* * *} \\
(1.22,1.53)\end{array}$ & $\begin{array}{c}1.39 * * * \\
(1.26,1.54)\end{array}$ & $\begin{array}{c}1.42 * * * \\
(1.28,1.58)\end{array}$ & $\begin{array}{c}1.30^{* * * *} \\
(1.13,1.50)\end{array}$ & $\begin{array}{c}1.13 \\
(0.94,1.36)\end{array}$ & $\begin{array}{c}1.44^{* * *} \\
(1.31,1.59)\end{array}$ \\
\hline Too much computer work & $\begin{array}{c}1.15^{*} \\
(1.03,1.28)\end{array}$ & $\begin{array}{c}1.33^{* * *} \\
(1.19,1.48)\end{array}$ & $\begin{array}{c}1.27^{* * *} \\
(1.14,1.41)\end{array}$ & $\begin{array}{c}1.23^{* * *} \\
(1.07,1.42)\end{array}$ & $\begin{array}{c}1.21^{*} \\
(1.02,1.44)\end{array}$ & $\begin{array}{c}1.27^{* * *} \\
(1.14,1.42)\end{array}$ \\
\hline Lack of training on new equipment & $\begin{array}{c}1.25^{* * *} \\
(1.14,1.38)\end{array}$ & $\begin{array}{c}1.31^{* * *} \\
(1.20,1.43)\end{array}$ & $\begin{array}{c}1.47^{* * *} \\
(1.33,1.63)\end{array}$ & $\begin{array}{c}1.24^{* * *} \\
(1.09,1.41)\end{array}$ & $\begin{array}{c}1.09 \\
(0.91,1.27)\end{array}$ & $\begin{array}{c}1.40^{* * *} \\
(1.27,1.54)\end{array}$ \\
\hline Perceived pressure to volunteer free time & $\begin{array}{c}1.25^{* * *} \\
(1.12,1.39) \\
\end{array}$ & $\begin{array}{c}1.26^{* * *} \\
(1.14,1.40) \\
\end{array}$ & $\begin{array}{c}1.24^{* * *} \\
(1.12,1.37) \\
\end{array}$ & $\begin{array}{c}1.21^{* * *} \\
(1.07,1.38) \\
\end{array}$ & $\begin{array}{c}1.01 \\
(0.84,1.21) \\
\end{array}$ & $\begin{array}{c}1.25^{* * *} \\
(1.12,1.40) \\
\end{array}$ \\
\hline Dealing with supervisors & $\begin{array}{c}1.39^{* * *} \\
(1.24,1.55)\end{array}$ & $\begin{array}{c}1.54^{* * *} \\
(1.38,1.71)\end{array}$ & $\begin{array}{c}1.50 * * * \\
(1.34,1.68)\end{array}$ & $\begin{array}{c}1.50 * * * \\
(1.29,1.75)\end{array}$ & $\begin{array}{c}1.14 \\
(0.95,1.36)\end{array}$ & $\begin{array}{c}1.52 * * * \\
(1.37,1.69)\end{array}$ \\
\hline Inconsistent leadership style & $\begin{array}{c}1.34^{* * *} \\
(1.20,1.50)\end{array}$ & $\begin{array}{c}1.48^{* * *} \\
(1.33,1.65)\end{array}$ & $\begin{array}{c}1.42 * * * \\
(1.27,1.58)\end{array}$ & $\begin{array}{c}1.44^{* * *} \\
(1.22,1.69)\end{array}$ & $\begin{array}{c}1.17 \\
(0.96,1.41)\end{array}$ & $\begin{array}{c}1.48^{* * *} \\
(1.34,1.63)\end{array}$ \\
\hline Lack of resources & $\begin{array}{c}1.41^{* * *} \\
(1.26,1.57) \\
\end{array}$ & $\begin{array}{c}1.40^{* * *} \\
(1.27,1.55) \\
\end{array}$ & $\begin{array}{c}1.40^{* * *} \\
(1.26,1.55) \\
\end{array}$ & $\begin{array}{c}1.31^{* * *} \\
(1.14,1.52) \\
\end{array}$ & $\begin{array}{c}1.13 \\
(0.94,1.35) \\
\end{array}$ & $\begin{array}{c}1.55^{* * *} \\
(1.40,1.71) \\
\end{array}$ \\
\hline Unequal sharing of work responsibilities & $\begin{array}{c}1.29 * * * \\
(1.17,1.43)\end{array}$ & $\begin{array}{c}1.43^{* * *} \\
(1.30,1.57)\end{array}$ & $\begin{array}{c}1.41^{* * *} \\
(1.28,1.56)\end{array}$ & $\begin{array}{c}1.30 * * * \\
(1.14,1.49)\end{array}$ & $\begin{array}{c}1.25 * \\
(1.05,1.49)\end{array}$ & $\begin{array}{c}1.44^{* * *} \\
(1.31,1.58)\end{array}$ \\
\hline $\begin{array}{l}\text { If you are sick or injured your co-workers seem } \\
\text { to look down on you }\end{array}$ & $\begin{array}{c}1.28^{* * *} \\
(1.18,1.40)\end{array}$ & $\begin{array}{c}1.43^{* * *} \\
(1.32,1.56)\end{array}$ & $\begin{array}{c}1.38^{* * *} \\
(1.26,1.50)\end{array}$ & $\begin{array}{c}1.62 * * * \\
(1.43,1.85)\end{array}$ & $\begin{array}{c}1.25^{* *} \\
(1.08,1.45)\end{array}$ & $\begin{array}{c}1.46^{* * *} \\
(1.33,1.60)\end{array}$ \\
\hline
\end{tabular}


Table 3. Cont

\begin{tabular}{|c|c|c|c|c|c|c|}
\hline \multirow{2}{*}{ Occupational Stressor } & PTSD $^{a}$ & $\begin{array}{c}\text { Major Depressive } \\
\text { Disorder }\end{array}$ & $\begin{array}{l}\text { Generalized } \\
\text { Anxiety }\end{array}$ & Panic Disorder & Alcohol Use Disorder ${ }^{b}$ & Any Mental Disorder \\
\hline & $\begin{array}{c}\text { AOR } \\
(95 \% \mathrm{CI})\end{array}$ & $\begin{array}{c}\text { AOR } \\
(95 \% \text { CI })\end{array}$ & $\begin{array}{c}\text { AOR } \\
(95 \% \text { CI })\end{array}$ & $\begin{array}{c}\text { AOR } \\
(95 \% \mathrm{CI})\end{array}$ & $\begin{array}{c}\text { AOR } \\
(95 \% \mathrm{CI})\end{array}$ & $\begin{array}{c}\text { AOR } \\
(95 \% \mathrm{CI})\end{array}$ \\
\hline Internal investigations & $\begin{array}{c}1.31^{* * *} \\
(1.20,1.42)\end{array}$ & $\begin{array}{c}1.28^{* * *} \\
(1.18,1.38)\end{array}$ & $\begin{array}{c}1.22 * * * \\
(1.12,1.32)\end{array}$ & $\begin{array}{c}1.24^{* * *} \\
(1.11,1.38)\end{array}$ & $\begin{array}{c}1.12 \\
(0.97,1.29)\end{array}$ & $\begin{array}{c}1.28^{* * *} \\
(1.18,1.39)\end{array}$ \\
\hline Dealing with the court system & $\begin{array}{c}1.24^{* * *} \\
(1.12,1.38)\end{array}$ & $\begin{array}{c}1.38^{* * *} \\
(1.25,1.53)\end{array}$ & $\begin{array}{c}1.28^{* * *} \\
(1.15,1.41)\end{array}$ & $\begin{array}{c}1.39 * * * \\
(1.22,1.59)\end{array}$ & $\begin{array}{c}1.15 \\
(0.97,1.37)\end{array}$ & $\begin{array}{c}1.32^{* * *} \\
(1.19,1.47)\end{array}$ \\
\hline The need to be accountable for doing your job & $\begin{array}{c}1.33^{* * *} \\
(1.20,1.47)\end{array}$ & $\begin{array}{c}1.35^{* * *} \\
(1.23,1.48)\end{array}$ & $\begin{array}{c}1.36^{* * *} \\
(1.23,1.49)\end{array}$ & $\begin{array}{c}1.39 * * * \\
(1.21,1.59)\end{array}$ & $\begin{array}{c}1.03 \\
(0.87,1.21)\end{array}$ & $\begin{array}{c}1.37 * * * \\
(1.24,1.50)\end{array}$ \\
\hline Inadequate equipment & $\begin{array}{c}1.27^{* * *} \\
(1.16,1.40)\end{array}$ & $\begin{array}{c}1.33^{* * *} \\
(1.22,1.45)\end{array}$ & $\begin{array}{c}1.27 * * * \\
(1.16,1.39)\end{array}$ & $\begin{array}{c}1.24^{* * *} \\
(1.10,1.40)\end{array}$ & $\begin{array}{c}1.05 \\
(0.90,1.23) \\
\end{array}$ & $\begin{array}{c}1.39 * * * \\
(1.27,1.53) \\
\end{array}$ \\
\hline Total Mean Organizational Stress Score & $\begin{array}{c}2.10^{* * *} \\
(1.75,2.53)\end{array}$ & $\begin{array}{c}2.63^{* * *} \\
(2.17,3.19)\end{array}$ & $\begin{array}{c}2.54^{* * *} \\
(2.08,3.09)\end{array}$ & $\begin{array}{c}2.51 * * * \\
(1.92,3.28)\end{array}$ & $\begin{array}{c}1.45^{*} \\
(1.08,1.93)\end{array}$ & $\begin{array}{c}2.62 * * * \\
(2.17,3.15)\end{array}$ \\
\hline \multicolumn{7}{|l|}{ Operational Stressors (PSP-Op) } \\
\hline Shift work & $\begin{array}{c}1.27^{* * *} \\
(1.15,1.41)\end{array}$ & $\begin{array}{c}1.28^{* * *} \\
(1.16,1.41)\end{array}$ & $\begin{array}{c}1.37^{* * *} \\
(1.24,1.53)\end{array}$ & $\begin{array}{c}1.33^{* * *} \\
(1.16,1.52)\end{array}$ & $\begin{array}{c}1.17^{*} \\
(1.01,1.36)\end{array}$ & $\begin{array}{c}1.38^{* * *} \\
(1.25,1.53)\end{array}$ \\
\hline Working alone at night & $\begin{array}{c}1.25^{* * *} \\
(1.13,1.38)\end{array}$ & $\begin{array}{c}1.21 * * * \\
(1.10,1.33)\end{array}$ & $\begin{array}{c}1.23 * * * \\
(1.12,1.35)\end{array}$ & $\begin{array}{c}1.23^{* * *} \\
(1.09,1.38)\end{array}$ & $\begin{array}{c}1.06 \\
(0.90,1.24)\end{array}$ & $\begin{array}{c}1.27 * * * \\
(1.15,1.41)\end{array}$ \\
\hline Over-time demands & $\begin{array}{c}1.17^{* * *} \\
(1.06,1.29) \\
\end{array}$ & $\begin{array}{c}1.32^{* * *} \\
(1.20,1.45) \\
\end{array}$ & $\begin{array}{c}1.30^{* * *} \\
(1.18,1.42) \\
\end{array}$ & $\begin{array}{c}1.18^{* * *} \\
(1.05,1.33) \\
\end{array}$ & $\begin{array}{c}1.20 * \\
(1.03,1.40) \\
\end{array}$ & $\begin{array}{c}1.37^{* * *} \\
(1.23,1.51) \\
\end{array}$ \\
\hline Risk of being injured on the job & $\begin{array}{c}1.37^{* * *} \\
(1.23,1.53)\end{array}$ & $\begin{array}{c}1.39 * * * \\
(1.25,1.53)\end{array}$ & $\begin{array}{c}1.49 * * * \\
(1.34,1.66)\end{array}$ & $\begin{array}{c}1.51 * * * \\
(1.31,1.75)\end{array}$ & $\begin{array}{c}1.24^{*} \\
(1.04,1.48)\end{array}$ & $\begin{array}{c}1.46^{* * *} \\
(1.32,1.63)\end{array}$ \\
\hline $\begin{array}{l}\text { Work-related activities on days off (e.g., court, } \\
\text { community events) }\end{array}$ & $\begin{array}{c}1.30^{* * *} \\
(1.16,1.46)\end{array}$ & $\begin{array}{c}1.33^{* * *} \\
(1.19,1.48)\end{array}$ & $\begin{array}{c}1.39^{* * * *} \\
(1.24,1.55)\end{array}$ & $\begin{array}{c}1.43^{* * *} \\
(1.25,1.64)\end{array}$ & $\begin{array}{c}1.09 \\
(0.90,1.32)\end{array}$ & $\begin{array}{c}1.35^{* * *} \\
(1.19,1.53)\end{array}$ \\
\hline $\begin{array}{l}\text { Traumatic events (e.g., motor vehicle accidents, } \\
\text { domestics, death, injury) }\end{array}$ & $\begin{array}{c}1.32 * * * \\
(1.20,1.46) \\
\end{array}$ & $\begin{array}{c}1.33^{* * *} \\
(1.22,1.46) \\
\end{array}$ & $\begin{array}{c}1.45^{* * *} \\
(1.32,1.59) \\
\end{array}$ & $\begin{array}{c}1.51^{* * *} \\
(1.33,1.71) \\
\end{array}$ & $\begin{array}{c}1.19^{*} \\
(1.01,1.40) \\
\end{array}$ & $\begin{array}{c}1.48^{* * *} \\
(1.33,1.64) \\
\end{array}$ \\
\hline Managing your social life outside of work & $\begin{array}{c}1.46^{* * *} \\
(1.31,1.64)\end{array}$ & $\begin{array}{c}1.86^{* * *} \\
(1.65,2.10)\end{array}$ & $\begin{array}{c}2.00 * * * \\
(1.76,2.28)\end{array}$ & $\begin{array}{c}1.76^{* * *} \\
(1.50,2.06)\end{array}$ & $\begin{array}{c}1.40^{* * *} \\
(1.17,1.68)\end{array}$ & $\begin{array}{c}1.80^{* * *} \\
(1.60,2.04)\end{array}$ \\
\hline $\begin{array}{l}\text { Not enough time available to spend with friends } \\
\text { and family }\end{array}$ & $\begin{array}{c}1.36^{* * *} \\
(1.23,1.51)\end{array}$ & $\begin{array}{c}1.49^{* * *} \\
(1.36,1.65)\end{array}$ & $\begin{array}{c}1.64^{* * *} \\
(1.47,1.82)\end{array}$ & $\begin{array}{c}1.46^{* * *} \\
(1.27,1.67)\end{array}$ & $\begin{array}{c}1.27^{* *} \\
(1.07,1.50)\end{array}$ & $\begin{array}{c}1.65^{* * *} \\
(1.49,1.83)\end{array}$ \\
\hline Paperwork & $\begin{array}{c}1.21^{* * *} \\
(1.09,1.34)\end{array}$ & $\begin{array}{c}1.34^{* * *} \\
(1.21,1.47)\end{array}$ & $\begin{array}{c}1.34^{* * *} \\
(1.21,1.48)\end{array}$ & $\begin{array}{c}1.23^{* * *} \\
(1.08,1.40)\end{array}$ & $\begin{array}{c}1.23 * \\
(1.04,1.44)\end{array}$ & $\begin{array}{c}1.32^{* * *} \\
(1.20,1.46)\end{array}$ \\
\hline
\end{tabular}


Table 3. Cont.

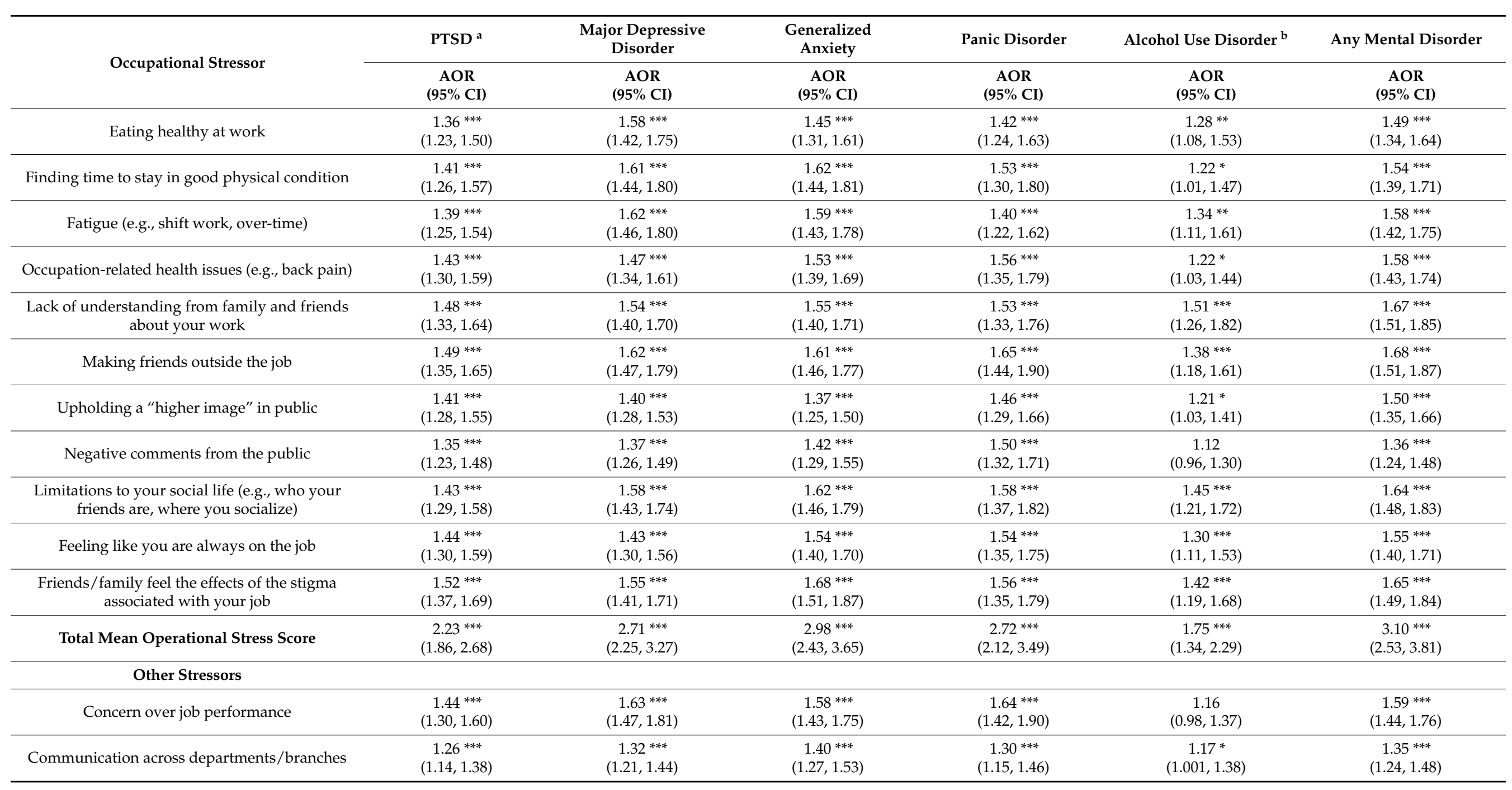


Table 3. Cont.

\begin{tabular}{|c|c|c|c|c|c|c|}
\hline \multirow{2}{*}{ Occupational Stressor } & PTSD $^{a}$ & $\begin{array}{c}\text { Major Depressive } \\
\text { Disorder }\end{array}$ & $\begin{array}{l}\text { Generalized } \\
\text { Anxiety }\end{array}$ & Panic Disorder & Alcohol Use Disorder ${ }^{b}$ & Any Mental Disorder \\
\hline & $\begin{array}{c}\text { AOR } \\
(95 \% \mathrm{CI})\end{array}$ & $\begin{array}{c}\text { AOR } \\
(95 \% \mathrm{CI})\end{array}$ & $\begin{array}{c}\text { AOR } \\
(95 \% \mathrm{CI})\end{array}$ & $\begin{array}{c}\text { AOR } \\
(95 \% \mathrm{CI})\end{array}$ & $\begin{array}{c}\text { AOR } \\
(95 \% \mathrm{CI})\end{array}$ & $\begin{array}{c}\text { AOR } \\
(95 \% \mathrm{CI})\end{array}$ \\
\hline Total Mean Other Stressors Score & $\begin{array}{c}1.65^{* * *} \\
(1.45,1.89)\end{array}$ & $\begin{array}{c}1.84^{* * *} \\
(1.61,2.09)\end{array}$ & $\begin{array}{c}1.96^{* * *} \\
(1.71,2.26)\end{array}$ & $\begin{array}{c}1.91^{* * *} \\
(1.59,2.30)\end{array}$ & $\begin{array}{c}1.28^{*} \\
(1.03,1.59)\end{array}$ & $\begin{array}{c}1.87^{* * *} \\
(1.63,2.14)\end{array}$ \\
\hline \multicolumn{7}{|c|}{$\begin{array}{l}\text { Notes. PTSD = post-traumatic stress disorder; AOR }=\text { odds ratio adjusted for sex, age, marital status, education, total years of service, total number of trauma exposures, and correctional worker occupational } \\
\text { category. a For PTSD models, age was collapsed into } 4 \text { age categories (i.e., } 20-29 \text { years, } 30-39 \text { years, } 40-49 \text { years, and } 50 \text { years and older). }{ }^{b} \text { For alcohol use models, age was collapsed into four age categories (i.e., } \\
20-29 \text { years, } 30-39 \text { years, } 40-49 \text { years, and } 50 \text { years and older). We were also unable to adjust for occupational category due to the low prevalence of screening positive for an alcohol use disorder in some of the } \\
\text { occupational categories. }{ }^{*} p<0.05 ;{ }^{* *} p<0.01 ; * * * p<0.001 .\end{array}$} \\
\hline & PTSD $^{a}$ & $\begin{array}{l}\text { Major Depressive } \\
\text { Disorder }\end{array}$ & Generalized Anxiety & Panic Disorder & Alcohol Use Disorder ${ }^{b}$ & Any Mental Disorder \\
\hline & $\begin{array}{c}\text { AOR } \\
(95 \% \mathrm{CI})\end{array}$ & $\begin{array}{c}\text { AOR } \\
(95 \% \mathrm{CI})\end{array}$ & $\begin{array}{c}\text { AOR } \\
(95 \% \mathrm{CI})\end{array}$ & $\begin{array}{c}\text { AOR } \\
(95 \% \mathrm{CI})\end{array}$ & $\begin{array}{c}\text { AOR } \\
(95 \% \mathrm{CI})\end{array}$ & $\begin{array}{c}\text { AOR } \\
(95 \% \mathrm{CI})\end{array}$ \\
\hline Total Number of Traumatic Exposures & $\begin{array}{c}1.17^{* * *} \\
(1.11,1.23)\end{array}$ & $\begin{array}{c}1.09 * * * \\
(1.05,1.14)\end{array}$ & $\begin{array}{c}1.07^{* *} \\
(1.03,1.12)\end{array}$ & $\begin{array}{c}1.10^{* *} \\
(1.04,1.18)\end{array}$ & $\begin{array}{c}1.00 \\
(0.93,1.09)\end{array}$ & $\begin{array}{c}1.09 * * * \\
(1.04,1.13)\end{array}$ \\
\hline \multicolumn{7}{|l|}{ Model 2} \\
\hline Mean Organizational Stress Score & $\begin{array}{c}1.40 * * \\
(1.10,1.79)\end{array}$ & $\begin{array}{c}1.49^{* * *} \\
(1.18,1.88)\end{array}$ & $\begin{array}{c}1.20 \\
(0.93,1.54)\end{array}$ & $\begin{array}{c}1.14 \\
(0.79,1.65)\end{array}$ & $\begin{array}{c}1.02 \\
(0.67,1.53)\end{array}$ & $\begin{array}{c}1.42 * * \\
(1.13,1.79)\end{array}$ \\
\hline Mean Operational Stress Score & $\begin{array}{c}1.84^{* * *} \\
(1.46,2.34)\end{array}$ & $\begin{array}{c}2.14 * * * \\
(1.71,2.69)\end{array}$ & $\begin{array}{c}2.27^{* * *} \\
(1.79,2.89)\end{array}$ & $\begin{array}{c}2.08^{* * *} \\
(1.49,2.89)\end{array}$ & $\begin{array}{c}1.77^{* *} \\
(1.22,2.58)\end{array}$ & $\begin{array}{c}2.43^{* * *} \\
(1.91,3.09)\end{array}$ \\
\hline Mean Other Stressors Score & $\begin{array}{c}1.05 \\
(0.88,1.25)\end{array}$ & $\begin{array}{c}1.01 \\
(0.85,1.19)\end{array}$ & $\begin{array}{c}1.13 \\
(0.95,1.35)\end{array}$ & $\begin{array}{c}1.17 \\
(0.92,1.48)\end{array}$ & $\begin{array}{c}0.84 \\
(0.64,1.12)\end{array}$ & $\begin{array}{c}0.99 \\
(0.83,1.18)\end{array}$ \\
\hline
\end{tabular}


Table 4. Cont.

\begin{tabular}{|c|c|c|c|c|c|c|}
\hline & PTSD $^{a}$ & $\begin{array}{c}\text { Major Depressive } \\
\text { Disorder }\end{array}$ & Generalized Anxiety & Panic Disorder & Alcohol Use Disorder ${ }^{b}$ & Any Mental Disorder \\
\hline & $\begin{array}{c}\text { AOR } \\
(95 \% \text { CI })\end{array}$ & $\begin{array}{c}\text { AOR } \\
(95 \% \text { CI })\end{array}$ & $\begin{array}{c}\text { AOR } \\
(95 \% \text { CI })\end{array}$ & $\begin{array}{c}\text { AOR } \\
(95 \% \text { CI })\end{array}$ & $\begin{array}{c}\text { AOR } \\
(95 \% \text { CI })\end{array}$ & $\begin{array}{c}\text { AOR } \\
(95 \% \text { CI })\end{array}$ \\
\hline Total Number of Traumatic Exposures & $\begin{array}{c}1.11^{* * *} \\
(1.04,1.17)\end{array}$ & $\begin{array}{c}1.00 \\
(0.95,1.05)\end{array}$ & $\begin{array}{c}0.97 \\
(0.92,1.03)\end{array}$ & $\begin{array}{c}1.01 \\
(0.94,1.09)\end{array}$ & $\begin{array}{c}0.94 \\
(0.86,1.02)\end{array}$ & $\begin{array}{c}1.00 \\
(0.95,1.05)\end{array}$ \\
\hline Mean Organizational Stress Score & $\begin{array}{c}1.34^{*} \\
(1.03,1.76)\end{array}$ & $\begin{array}{c}1.64^{* * *} \\
(1.26,2.12)\end{array}$ & $\begin{array}{c}1.29 \\
(0.92,1.03)\end{array}$ & $\begin{array}{c}1.29 \\
(0.86,1.95)\end{array}$ & $\begin{array}{c}0.93 \\
(0.58,1.50)\end{array}$ & $\begin{array}{c}1.55^{* * *} \\
(1.20,2.00)\end{array}$ \\
\hline Mean Operational Stress Score & $\begin{array}{c}1.74^{* * *} \\
(1.34,2.26)\end{array}$ & $\begin{array}{c}1.93^{* * *} \\
(1.51,2.48)\end{array}$ & $\begin{array}{c}2.24^{* * *} \\
(1.71,2.92)\end{array}$ & $\begin{array}{c}2.00^{* * *} \\
(1.39,2.89)\end{array}$ & $\begin{array}{c}1.99^{* *} \\
(1.29,3.05)\end{array}$ & $\begin{array}{c}2.37^{* * *} \\
(1.81,3.12)\end{array}$ \\
\hline Mean Other Stressors Score & $\begin{array}{c}1.07 \\
(0.88,1.30) \\
\end{array}$ & $\begin{array}{c}1.06 \\
(0.88,1.28) \\
\end{array}$ & $\begin{array}{c}1.20 \\
(0.98,1.46) \\
\end{array}$ & $\begin{array}{c}1.21 \\
(0.92,1.59) \\
\end{array}$ & $\begin{array}{c}0.90 \\
(0.65,1.24) \\
\end{array}$ & $\begin{array}{c}0.99 \\
(0.81,1.21) \\
\end{array}$ \\
\hline \multicolumn{7}{|l|}{ Model 4} \\
\hline Trauma Exposure by Organizational Stress Interaction Term & $\begin{array}{c}0.99 \\
(0.94,1.04)\end{array}$ & $\begin{array}{c}1.05^{*} \\
(1.01,1.09)\end{array}$ & $\begin{array}{c}1.00 \\
(0.96,1.05)\end{array}$ & $\begin{array}{c}1.03 \\
(0.97,1.09)\end{array}$ & $\begin{array}{c}0.98 \\
(0.91,1.04)\end{array}$ & $\begin{array}{c}1.02 \\
(0.97,1.07)\end{array}$ \\
\hline Trauma Exposure by Operational Stress Interaction Term & $\begin{array}{c}0.96 \\
(0.92,1.01)\end{array}$ & $\begin{array}{c}1.03 \\
(0.99,1.07)\end{array}$ & $\begin{array}{c}1.01 \\
(0.97,1.05)\end{array}$ & $\begin{array}{c}1.00 \\
(0.96,1.05)\end{array}$ & $\begin{array}{c}0.96 \\
(0.91,1.02)\end{array}$ & $\begin{array}{c}1.02 \\
(0.97,1.06)\end{array}$ \\
\hline
\end{tabular}

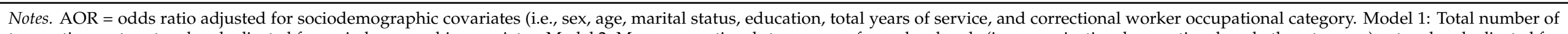

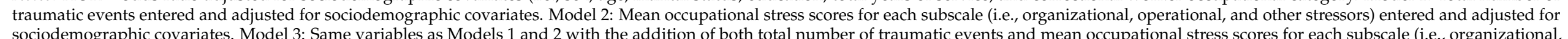

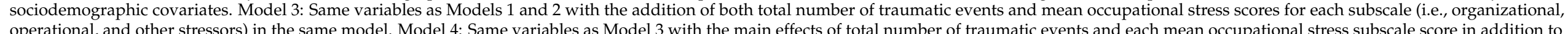

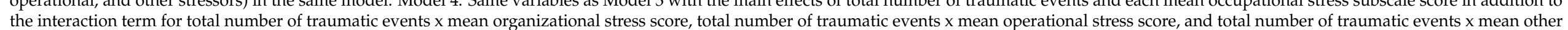

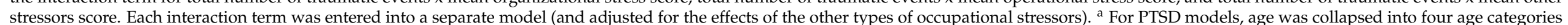

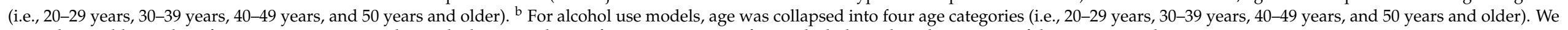
were also unable to adjust for occupation category due to the low prevalence of screening positive for an alcohol use disorder in some of the occupational categories. ${ }^{*} p<0.05 ;{ }^{* *} p<0.01$; ${ }^{* * *} p<0.001$. 


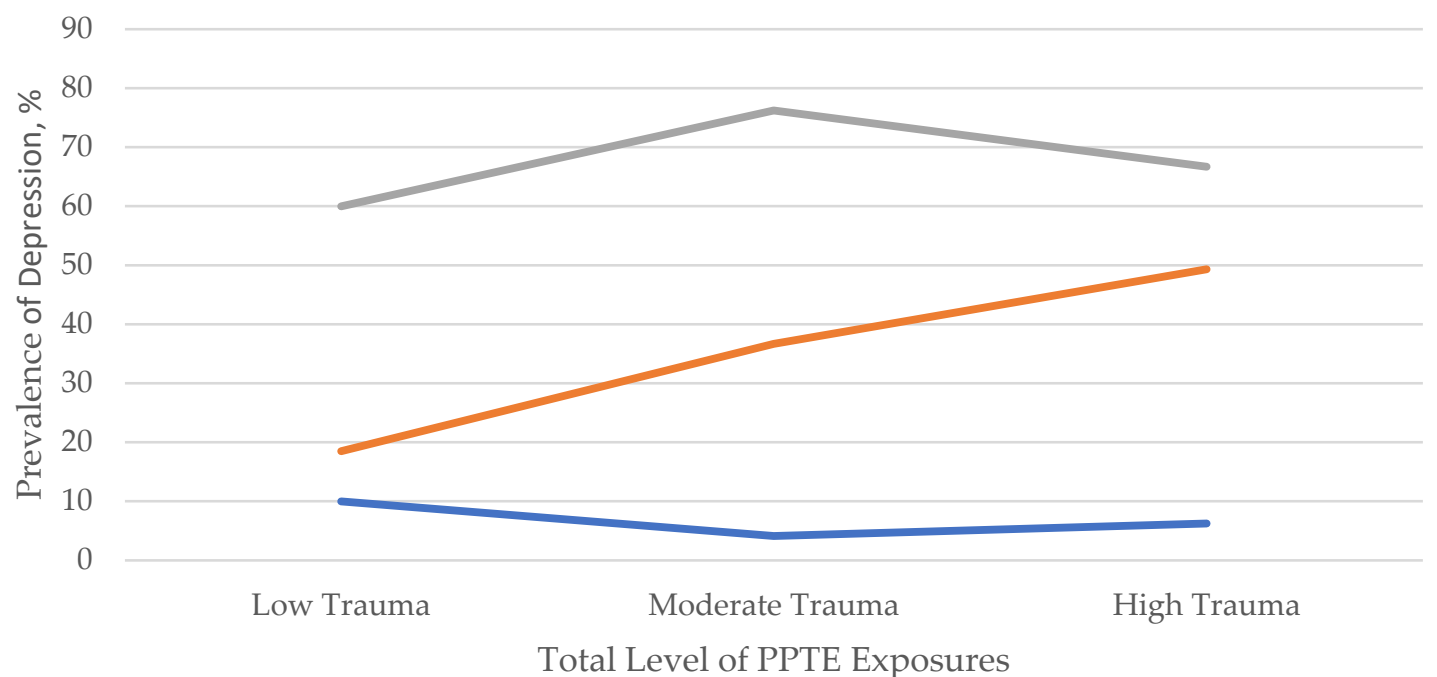

$\longrightarrow$ Low Organizational Stress $\longrightarrow$ Moderate Organizational Stress $\longrightarrow$ High Organizational Stress

Figure 1. Interaction of Total Trauma by Organizational Stress on Major Depressive Disorder Symptoms. Notes. Low trauma exposure and low organizational stress were defined as scores less than 1 standard deviation below their respective means; moderate trauma exposure and moderate organizational stress were defined as scores ranging from 1 standard deviation below the mean and 1 standard deviation above the mean; high levels of trauma exposure and high organizational stress were defined as scores more than 1 standard deviation above their respective means.

\section{Discussion}

The current study findings reveal that the occupational stressors associated with $\mathrm{CW}$ mental health are multifactorial thereby emphasizing the importance of evaluating numerous stressors in a single study to better understand which stressors are most salient within a given context. Results indicate that PPTE exposures remain significantly associated with PTSD among CWs, but daily occupational stressors account for substantial variance in mental health. Exposure to PPTEs in the workplace cannot be ignored but may prove more challenging as an area for intervention (i.e., the prevention of PPTEs); our results suggest that there are numerous other stressors that require attention in efforts to improve $\mathrm{CW}$ mental health. Based on our study's findings, interpersonal stressors, resource allocation problems, and struggles tied to one's well-being outside the walls of work are areas of particular significance for CW mental health.

Prior research on job stress within correctional work left unanswered questions about how occupational stressors may impact employee mental health and well-being. Using a concept as broad as job stress captures a "variety of negative, affective states" [72] (p. 513), but also leads to ambiguous and indeterminate implications of high job stress amongst CWs. In the current study, by shifting the dependent variable from job stress to mental health disorders we improve our understanding of the psychological challenges (e.g., PTSD, depression) experienced by CWs. In our sample, $29.7 \%$ screened positive for PTSD and $37.1 \%$ for Major Depressive Disorder, thereby highlighting the need for identification of the occupational stressors most relevant to the development of mental health disorders. Reports of CWs experiencing diverse occupational stressors (i.e., organizational and operational) indicated all stressors were associated with potentially problematic mental health symptoms. In other words, the outcomes associated with working in highly stressful environments with regular PPTE exposures appear to broadly increase risks for PTSIs.

The correctional occupational environment variables that influence mental health are varied and nuanced, which means more information is needed to effectively intervene 
to protect CWs. Using the PSQ-Org and PSQ-Op [56] and assessing diverse stressors including but not limited to roles (e.g., supervision, work-family conflict) and PPTE (e.g., fear of victimization, workplace violence) produced important novel results. Specifically, the occupational stressors most associated with mental health challenges for CWs (e.g., staff shortages, bureaucratic red tape, feeling that different rules apply to different people, lack of resources, constant changes in policy and legislation, dealing with co-workers) have not typically been the focus of previous research on CW well-being. This finding underlines the importance of including multiple stressors within a single study to determine which stressors are most impactful on CW mental health and therefore essential areas to target for intervention. Many of the stressors associated with the highest mean levels of stress in our study (e.g., inconsistent leadership style; not enough time available to spend with friends and family; risk of being injured on the job) were also consistent with earlier PSP research [15] as well as previous research specific to CW stress (e.g., quality supervision; work-family conflict; fear of victimization) [21].

All occupational stressors in the current study were associated with increased odds of a positive screen for PTSD, MDD, GAD, panic disorder, or any mental disorder, which further underscores previous results that PPTE impacts are idiosyncratic and potentially mitigated by occupational interventions [15], while further challenging the notions that only exposures to PPTEs warrant support. Furthermore, organizational stressors may account for more variance in mental health for PSP than operational stressors. Identifying pertinent organizational stressors elucidates tiered target areas for intervention and improvement from leadership, echoes results from the Canadian PSP study [15], and highlights the need for similar workplace improvements at the national and provincial levels. The importance of all occupational stressors included in the current study reveals both the vast potential for workplace improvements and also the opportunity to tier interventions, starting with the most salient stressors for CWs. Ontario CWs identified organizational stressors similar to those experienced by other PSP across Canada, which affords correctional leadership the opportunity to adopt successful interventions from other PSP workplaces. The organizational stressors associated with the highest mean levels of stress demonstrate a need for increased or more efficient resource allocations (e.g., staff shortages, lack of resources), as well as consistent and enhanced communication strategies from management and amongst employees (e.g., inconsistent leadership style, dealing with co-workers). Previous research supports a relationship between workplace interpersonal relationships and $\mathrm{CW}$ job stress $[73,74]$. Given the stress burden associated with constant changes to policy and legislation and the foreseeable reality that policies will continue to change, correctional leadership may want to explore alternative ways to roll out policies that could be more supportive to the psychological distress that CWs feel during transition periods. Additionally, where possible, CWs should be offered a forum to provide input into the development of policies that will impact their role.

The most pertinent organizational stressors remained consistent across the various correctional occupational categories, except for probation/parole officers who identified burdens in their role due to excessive administrative and computer work as the stressors associated with the highest mean levels of stress. The operational stressors reported as most concerning were connected to maintaining overall well-being and balancing work life and personal life (e.g., finding time to stay in good physical condition, fatigue, occupationrelated health issues, not enough time available to spend with friends and family, eating healthy at work). Additionally, the inability to manage a social life outside of work had the largest association with symptoms of MDD, GAD, panic disorder, and indications of any mental disorder. Helping to minimize the impact of work on maintaining a social life will require creative leadership interventions but remains an important aspect of sustainable mental health $[75,76]$. Despite the challenges associated with intervening on issues such as CW fatigue and CWs not finding enough time to spend with family and friends, the current results suggest that exploring innovative strategies to support employee wellbeing and work-life balance may help promote improved CW mental health. For example, 
institutions could incorporate onsite fitness equipment or break rooms with full kitchen facilities alongside longer lunch breaks, all of which can help promote improved well-being. Structural strategies may be challenging to implement, but offer potential pathways to support CW mental health and employee retention.

The mean levels of stress associated with working with the prisoner/probationer/parolee population were lower than the most stressful events identified in both organizational and occupational categories. The stressors identified as most challenging by participants highlight the challenges embedded within the broader correctional occupational environment and culture. The current results demonstrate how PPTE exposures interact with occupational stressors to influence risk for diverse mental health challenges. Our results reinforce the important role the occupational environment can play in employee mental health. Correctional occupational stressors may facilitate dysfunctional workplace behaviour, declined health, and negative personality changes [77,78]. Optimistically, many of the stressors identified in our study are also opportunities for workplaces to better protect CW mental health. In line with previous research results with diverse Canadian PSP, PPTE exposures are not the only contributing workplace factor to employee mental health [15] and may be less salient than occupational stressors. There appear to be several stressors that can be modified by the organization to support a psychologically healthier workforce better able to manage and cope with PPTE exposures common to correctional environments (e.g., violence, overdoses, suicide attempts). Given limited resources, it is essential that interventions to improve employee psychological wellbeing are grounded in evidence that supports both the target area of change and the efficacy of a proposed intervention. The results from our study elucidate the most prominent stressors for Ontario CWs and provide policymakers and correctional management a potential starting point for affecting changes related to employee psychological well-being.

\section{Limitations}

The current study is limited by several factors, including that the CW sample was self-selected instead of stratified or random, which limits the representativeness and generalizability of our results. Survey responses remain anonymous, which leaves space for possible challenges tied to missing, biased, or erroneous data. We used screening measures for mental health assessments rather than diagnostic tools, which leads us to recommend future researchers employ clinical interviews, rather than screening tools, to provide diagnostic assessments that would make the results more robust. We used self-report to estimate the number of PPTE exposures, which involves challenges with participant recall. We did not assess the prevalence and impact of family-based stressors or individual difference variables, all of which should be assessed in future research to better understand the interactive effects on mental health and well-being. In addition, although the PSQ-Org and PSQ-Op are validated tools developed as two independent measures of occupational stressors, the three additional items (i.e., concern over job performance, communication across departments/branches, and working in close contact with the inmate/client population) used as an "other stressors" subscale in this study were researcher developed and not validated for use in this population. The low alpha coefficient (i.e., 0.71 ) for this subscale suggests that the reliability of our findings related to this subscale be interpreted with caution. Future researchers should also consider interaction effects between indirect and direct PPTE exposures with perceptions of occupational stress and mental health. Finally, the cross-sectional data prohibits assessments of risk and causality (i.e., we cannot analyze the order effects of PPTE exposures, occupational stressors, and mental health impacts). Future longitudinal studies should assess for the risk tied to diverse stressors, which are instrumental to determining strategies to support the mental health needs of CWs.

\section{Conclusions}

Earlier research on CW psychological well-being established statistically significant relationships between the correctional environment and employee experiences of stress. 
The current study builds on past research by estimating the relative impact of 43 different occupational stressors on $\mathrm{CW}$ mental health thereby presenting a roadmap for a tiered approach to fostering interventions targeting CW stress and mental health. PPTE exposures are likely to be a reality for CWs; however, interventions targeting organizational and operational stressors may help mitigate CWs experiencing mental health challenges. For example, reducing occupational stressors (e.g., increase leadership consistency, reduce procedural uncertainty) may help to mitigate PTSD symptoms. Based on the stressors identified as most salient by CWs in our study, there may be particularly important opportunities to improve mental health by increasing staffing levels, improving communication from management to staff and amongst staff, and supporting staff to develop a work-life balance that attends to their physical, mental, and social needs. Ideally, the development of interventions targeting occupational stressors should be accompanied by research that tests the effectiveness of these efforts to ensure that occupational interventions help improve the psychological well-being of CWs, making carceral environments safer and healthier workplaces.

Supplementary Materials: The following are available online at https:/ /www.mdpi.com/article/10 .3390/ijerph181910018/s1, Table S1: Sociodemographic Covariates for Respondents Included and Excluded in Occupational Stressors and Trauma Exposures Analyses.

Author Contributions: All authors made substantial contributions to the overall paper and reviewed and revised the paper as necessary. Conceptualization, R.R., R.N.C. and D.G.; methodology, R.N.C., T.T. and T.O.A.; formal analysis, T.T., T.O.A., R.N.C. and R.R.; investigation, R.R., R.N.C. and D.G.; resources, R.R. and D.G.; data curation, R.R.; writing—original draft preparation, K.K. and R.R.; writing-review and editing, K.K., R.R., R.N.C., T.T. and. T.O.A.; supervision, R.R., R.N.C. and T.O.A.; project administration, R.R.; funding acquisition, R.R. and D.G. All authors have read and agreed to the published version of the manuscript.

Funding: The author(s) disclosed receipt of the following financial support for the research, authorship, and/or publication of this article: R. Nicholas Carleton's research is supported by the Canadian Institutes of Health Research (CIHR) through a New Investigator Award (FRN: 13666). Tracie O. Afifi's research is supported by a Tier I Canada Research Chair. This research was also funded in part by a CIHR Catalyst Grant (FRN: 16234).

Institutional Review Board Statement: Prior to commencement the study was reviewed for ethical compliance by the Queen's University and Affiliated Health Sciences Centre Research Ethics Board (file \#6024787) as well as the Research Ethics Boards at both the University of Regina (file \#2017-098) and Memorial University of Newfoundland (file \#20201330-EX).

Informed Consent Statement: Informed consent was obtained from all subjects involved in the study.

Acknowledgments: We would like to extend our gratitude to all participants in the study, the Ministry of the Solicitor General of Ontario (particularly Tina Flood and Vicente Gannam), and the Ontario Public Service Employees Union.

Conflicts of Interest: The authors declare no conflict of interest. The funders had no role in the design of the study; in the collection, analyses, or interpretation of data; in the writing of the manuscript, or in the decision to publish the result.

\section{References}

1. Ricciardelli, R. Surviving Incarceration: Inside Canadian Prisons; Wilfrid Laurier University Press: Brantford, ON, Canada, 2014.

2. Pratt, J. Punishment and the civilizing process. In The SAGE Handbook of Punishment and Society; Simon, J., Sparks, R., Eds.; Sage Publications: London, UK, 2013.

3. Butler, D.; Tasca, M.; Zhang, Y.; Carpenter, C. A systematic and meta-analytic review of the literature on correctional officers: Identifying new avenues for research. J. Crim. Justice 2019, 60, 84-92. [CrossRef]

4. Ricciardelli, R. Also Serving Time: Canada's Provincial and Territorial Correctional Officers; University of Toronto Press: Toronto, ON, Canada, 2019. 
5. Ricciardelli, R. Recognizing Federal Correctional Officers as First Responders and under the Memorial Grant. A Position Paper for the Minister of Public Safety and Emergency Preparedness: The Honourable Ralph Goodale. 7 July 2019. Available online: https: / / ucco-sacc-csn.ca/assets/uploads/2019/08/08-2019-COs-as-First-Responders_Final.pdf (accessed on 16 September 2021).

6. Regehr, C.; Carey, M.; Wagner, S.; Alden, L.; Buys, N.; Corneil, W.; Fyfe, T.; Fraess-Phillips, A.; Krutop, E.; Matthews, L.; et al. Prevalence of PTSD, depression and anxiety disorders in correctional officers: A systematic review. Correct. Policy Pract. Res. 2021, 6, 229-241. [CrossRef]

7. Norman, M.; Ricciardelli, R. Operational and organisational stressors in community correctional work: Insights from probation and parole officers in Ontario, Canada. Probat. J. 2021, 0264550520984253. [CrossRef]

8. Lambert, E.; Minor, K.; Wells, J.; Hogan, N. Social support's relationship to correctional staff job stress, job involvement, job satisfaction, and organizational commitment. Soc. Sci. J. 2016, 53, 22-32. [CrossRef]

9. Canadian Institute of Public Safety Research and Treatment. Glossary of Terms: Psychologically Traumatic Event. Available online: https: / / www.cipsrt-icrtsp.ca/en/resources/glossary-of-terms\#667 (accessed on 3 September 2021).

10. Carleton, R.N.; Afifi, T.O.; Taillieu, T.; Turner, S.; Krakauer, R.; Anderson, G.S.; MacPhee, R.S.; Ricciardelli, R.; Cramm, H.A.; Groll, D.; et al. Exposures to potentially traumatic events among public safety personnel in Canada. Can. J. Behav. Sci. 2019, 51, 37-52. [CrossRef]

11. Denhof, M.D.; Spinaris, C.G. Prevalence of Trauma-Related Health Conditions in Correctional Officers: A Profile of Michigan Corrections Organization Members. 2016. Available online: https://www.mco-seiu.org/wp-content/uploads/2016/05/MCOPaper_FINAL.pdf (accessed on 16 September 2021).

12. James, L.; Todak, N. Prison employment and post-traumatic stress disorder: Risk and protective factors. Am. J. Ind. Med. 2018, 61, 725-732. [CrossRef]

13. Carleton, R.N.; Afifi, T.O.; Turner, S.; Taillieu, T.; Duranceau, S.; LeBouthillier, D.M.; Sareen, J.; Ricciardelli, R.; MacPhee, R.S.; Groll, D.; et al. Mental disorder symptoms among public safety personnel. Can. J. Psychiatry 2018, 63, 54-64. [CrossRef]

14. Carleton, R.N.; Ricciardelli, R.; Taillieu, T.; Mitchell, M.; Andres, E.; Afifi, T.O. Provincial correctional service workers: The prevalence of mental disorders. Int. J. Environ. Res. Public Health 2020, 17, 2203. [CrossRef] [PubMed]

15. Carleton, R.N.; Afifi, T.O.; Taillieu, T.; Turner, S.; Mason, J.; Ricciardelli, R.; McCreary, D.; Vaughan, A.; Anderson, G.; Krakauer, R.; et al. Assessing the relative impact of diverse stressors among public safety personnel. Int. J. Environ. Res. Public Health 2020, 17, 1234. [CrossRef]

16. Fusco, N.; Ricciardelli, R.; Jamshidi, L.; Carleton, R.N.; Barnim, N.; Hilton, Z.; Groll, D. When our work hits home: Trauma and mental disorders in correctional officers and other correctional workers. Front. Psychiatry 2021, 11, 493391. [CrossRef]

17. Jaegers, L.A.; Matthieu, M.M.; Vaughn, M.G.; Werth, P.; Katz, I.M.; Ahmad, S.O. Posttraumatic stress disorder and job burnout among jail officers. J. Occup. Environ. Med. 2019, 61, 505-510. [CrossRef] [PubMed]

18. Ricciardelli, R.; Groll, D.; Czarnuch, S.; Carleton, R.N.; Cramm, H. Behind the frontlines: Exploring the mental health and help-seeking behaviours of public safety personnel who work to support frontline operations. Annu. Rev. Interdiscip. Justice Res. 2019, 8, 315-348.

19. Oliphant, R.C. Healthy Minds, Safe Communities: Supporting Our Public Safety Officers through a National Strategy for Operational Stress Injuries; Security, Standing Committee on Public Safety and National Security, Ed.; Standing Committee on Public Safety and National Security: Ottawa, ON, Canada, 2016; Available online: http:/ / www.parl.gc.ca/HousePublications / Publication. aspx?DocId=8457704\&Language=E (accessed on 13 August 2021).

20. Picard, M. (Chair). National roundtable on post-traumatic stress disorder. In Proceedings of the Standing Committee on Public Safety and National Security (SECU), Regina, SK, Canada, 29 January 2016.

21. Lambert, E.; Hogan, N. Correctional staff: The issue of job stress. In The Practice of Correctional Psychology; Ternes, M., Magaletta, P., Patry, M., Eds.; Springer: Cham, Switzerland, 2018; pp. 259-281. [CrossRef]

22. Lambert, E.G.; Hogan, N.L.; Griffin, M.L.; Kelley, T. The correctional staff burnout literature. Crim. Justice Stud. 2015, 28 , 397-443. [CrossRef]

23. Arnold, H. The psychological and emotional effects of prison on prison staff. In The Routledge International Handbook of Forensic Psychology in Secure Settings; Ireland, J., Ireland, C., Fisher, M., Gredecki, N., Eds.; Routledge: London, UK, 2017 ; pp. $283-299$.

24. Armstrong, G.; Griffin, M. Does the job matter? Comparing correlates of stress among treatment and correctional staff in prisons. J. Crim. Justice 2004, 32, 577-592. [CrossRef]

25. Dowden, C.; Tellier, C. Predicting work-related stress in correctional officers: A meta- analysis. J. Crim. Justice 2004, 32, 31-47. [CrossRef]

26. Lambert, E.; Cluse-Tolar, T.; Hogan, N. This job is killing me: The impact of job characteristics on correctional staff job stress. Appl. Psychol. Crim. Justice 2007, 3, 117-142.

27. Armstrong, G.; Atkin-Plunk, C.; Wells, J. The relationship between work-family conflict, correctional officer job stress, and job satisfaction. Crim. Justice Behav. 2015, 42, 1066-1082. [CrossRef]

28. Liu, J.; Lambert, E.G.; Jiang, S.; Zhang, J. A research note on the association between work-family conflict and job stress among Chinese prison staff. Psychol. Crime Law 2017, 23, 633-646. [CrossRef]

29. Lambert, E.; Qureshi, H.; Frank, J.; Keena, L.; Hogan, N. The relationship of work-family conflict with job stress among Indian police officers: A research note. Police Pract. Res. 2017, 18, 37-48. [CrossRef] 
30. Obidoa, C.; Reeves, D.; Warren, N.; Reisine, S.; Cherniack, M. Depression and work family conflict among corrections officers. J. Occup. Environ. Med. 2011, 53, 1294-1301. [CrossRef]

31. Lambert, E.; Altheimer, I.; Hogan, N. An exploratory examination of a gendered model of the effects of role stressors. Women Crim. Justice 2010, 20, 193-217. [CrossRef]

32. Liu, J.; Lambert, I.E.; Kelley, T.; Zhang, J.; Jiang, S. Exploring association between work-family conflict and job involvement. Int. J. Offender Ther. Comp. Criminol. 2020, 64, 791-817. [CrossRef]

33. Keinan, G.; Malach-Pines, A. Stress and burnout among prison personnel: Sources, outcomes, and intervention strategies. Crim. Justice Behav. 2007, 34, 380-398. [CrossRef]

34. Paoline, E.; Lambert, E. The issue of control in jail: The effects of professionalism, detainee control, and administrative support on job stress, job satisfaction, and organizational commitment among jail staff. Am. J. Crim. Justice 2012, 37, 179-199. [CrossRef]

35. Paoline, E.; Lambert, E.; Hogan, N. Job stress and job satisfaction among jail staff: Exploring gendered effects. Women Crim. Justice 2015, 25, 339-359. [CrossRef]

36. Savicki, V.; Cooley, E.; Gjesvold, J. Harassment as a predictor of job burnout in correctional officers. Crim. Justice Behav. 2003, 30, 602-619. [CrossRef]

37. Ricciardelli, R.; Carleton, N.; Groll, D.; Cramm, H. Qualitatively unpacking Canadian public safety personnel experiences of trauma and their well-being. Can. J. Criminol. Crim. Justice 2018, 60, 566-577. [CrossRef]

38. Ricciardelli, R.; Power, N. How "conditions of confinement" impact "conditions of employment": The work-related well-being of provincial correctional officers in Atlantic Canada. Violence Vict. 2020, 35, 88-107. [CrossRef] [PubMed]

39. Union of Canadian Correctional Officers. Plus Que Jamais, l'Unité c'Est la Clé! More Than Ever, Unity Is the Key. Vigilance no 4. July 2016. Available online: https:/ / ucco-sacc-csn.ca/assets/uploads/2019/03/UCCO-SACC_Vigilance-SUmmer-2016.pdf (accessed on 16 September 2021).

40. Konda, S.; Tiesman, H.; Reichard, A.; Hartley, D.U.S. correctional officers killed or injured on the job. Correct. Today 2013, 75, 122-123. [PubMed]

41. Blevins, C.A.; Weathers, F.W.; Davis, M.T.; Witte, T.K.; Domino, J.L. The Posttraumatic Stress Disorder Checklist for DSM-5 (PCL-5): Development and initial psychometric evaluation. J. Trauma. Stress 2015, 28, 489-498. [CrossRef]

42. Weathers, F.W.; Litz, B.T.; Keane, T.M.; Palmieri, P.A.; Marx, B.P.; Schnurr, P.P. The PTSD Checklist for DSM-5 (PCL-5). Scale Available from the National Center for PTSD. Available online: https://www.ptsd.va.gov/professional/assessment/adult-sr/ ptsd-checklist.asp (accessed on 16 September 2021).

43. Spinaris, C.G.; Denhof, M.D.; Kellaway, J.A. Posttraumatic Stress Disorder in United States Corrections Professionals: Prevalence and Impact on Health and Functioning. 2012. Available online: https:/ / www.ojp.gov/ncjrs/virtual-library/abstracts/ posttraumatic-stress-disorder-united-states-corrections (accessed on 16 September 2021).

44. American Psychiatric Association. Diagnostic and Statistical Manual of Mental Disorders, 5th ed.; American Psychiatric Association: Washington, DC, USA, 2013.

45. Bremner, J.D. Does Stress Damage the Brain? Understanding Trauma-Related Disorders from a Mind-Body Perspective; W.W. Norton \& Co.: New York, NY, USA, 2002.

46. Andersen, L.; Hogh, A.; Elklit, A.; Andersen, J.; Biering, K. Work-related threats and violence and post-traumatic symptoms in four high-risk occupations: Short- and long- term symptoms. Int. Arch. Occup. Environ. Health 2019, 92, 195-208. [CrossRef] [PubMed]

47. Bourbonnais, R.; Jauvin, N.; Dussault, J.; Vézina, M. Psychosocial work environment, interpersonal violence at work and mental health among correctional officers. Int. J. Law Psychiatry 2007, 30, 355-368. [CrossRef] [PubMed]

48. Kunst, M.; Bogaerts, S.; Winkel, F.W. Peer and inmate aggression, type D-personality and posttraumatic stress among Dutch prison workers. Stress Health 2009, 25, 387-395. [CrossRef]

49. Kunst, M. Working in prisons: A critical review of stress in the occupation of correctional officers. In Handbook of Stress in the Occupations; Lnagan-Fox, J., Cooper, C., Eds.; Edward Elgar Publishing: Cheltenham, UK, 2011; pp. 241-283.

50. Bezerra, C.; Assis, S.; Constantino, P. Psychological distress and work stress in correctional officers: A literature review. Ciência E Saúde Coletiva 2016, 21, 2135-2146.

51. Neveu, J. Jailed resources: Conservation of resources theory as applied to burnout among prison guards. J. Organ. Behav. 2007, 28, 21-42. [CrossRef]

52. Boudoukha, A.; Hautekeete, M.; Abdelaoui, S.; Groux, W.; Garay, D. Burnout et victimisations: Effets des agressions des personnes détenues envers les personnels de surveillance. L'Encéphale 2011, 37, 284-292. [CrossRef]

53. Griffin, M.; Hogan, N.; Lambert, E. Doing "People Work" in the prison setting: An examination of the job characteristics model and correctional staff burnout. Crim. Justice Behav. 2012, 39, 1131-1147. [CrossRef]

54. Public Safety Canada. Post-Traumatic Stress Injuries and Support for Public Safety Officers. 2018. Available online: https: //www.publicsafety.gc.ca/cnt/mrgnc-mngmnt/mrgnc-prprdnss/ptsi-en.aspx (accessed on 16 September 2021).

55. Silliker, A. Correctional Officers Calling for Presumptive PTSD Coverage across Canada. Canadian Occupational Safety, pp. 14-17. February/March 2018. Available online: https:/ / www.cos-mag.com/psychological-health-safety/35965-correctionalofficers-calling-for-presumptive-ptsd-coverage-across-canada / (accessed on 16 September 2021).

56. McCreary, D.R.; Thompson, M.M. Development of two reliable and valid measures of stressors in policing: The operational and organizational police stress questionnaires. Int. J. Stress Manag. 2006, 13, 494-518. [CrossRef] 
57. Ashbaugh, A.R.; Houle-Johnson, S.; Herbert, C.; El-Hage, W.; Brunet, A. Psychometric validation of the English and French versions of the Posttraumatic Stress Disorder Checklist for DSM-5 (PCL-5). PLoS ONE 2016, 11, e0161645. [CrossRef] [PubMed]

58. Bovin, M.J.; Marx, B.P.; Weathers, F.W.; Gallagher, M.W.; Rodriguez, P.; Schnurr, P.P.; Keane, T.M. Psychometric properties of the PTSD Checklist for Diagnostic and Statistical Manual of Mental Disorders-Fifth Edition (PCL-5) in veterans. Psychol. Assess. 2016, 28, 1379-1391. [CrossRef]

59. MacIntosh, H.B.; Séguin, G.; Abdul-Ramen, I.; Randy, M. Première Traduction Française PCL-5-LEC, Civilian Checklist for PTSD, DSM5; McGill: Montreal, QC, Canada, 2015.

60. Beard, C.; Bjorgvinsson, T. Beyond generalized anxiety disorder: Psychometric properties of the GAD-7 in a heterogeneous psychiatric sample. J. Anxiety Disord. 2014, 28, 547-552. [CrossRef]

61. Kroenke, K.; Spitzer, R.L.; Williams, J.B. The PHQ-9: Validity of a brief depression severity measure. J. Gen. Intern. Med. 2001, 16, 606-613. [CrossRef] [PubMed]

62. Kroenke, K.; Spitzer, R.L.; Williams, J.B.; Lowe, B. The Patient Health Questionnaire Somatic, Anxiety, and Depressive Symptom Scales: A systematic review. Gen. Hosp. Psychiatry 2010, 32, 345-359. [CrossRef] [PubMed]

63. Lowe, B.; Unutzer, J.; Callahan, C.M.; Perkins, A.J.; Kroenke, K. Monitoring depression treatment outcomes with the patient health questionnaire-9. Med. Care 2004, 42, 1194-1201. [CrossRef]

64. Manea, L.; Gilbody, S.; McMillan, D. A diagnostic meta-analysis of the Patient Health Questionnaire-9 (PHQ-9) algorithm scoring method as a screen for depression. Gen. Hosp. Psychiatry 2015, 37, 67-75. [CrossRef]

65. Spitzer, R.L.; Kroenke, K.; Williams, J.B.; Lowe, B. A brief measure for assessing generalized anxiety disorder: The GAD-7. Arch. Intern. Med. 2006, 166, 1092-1097. [CrossRef] [PubMed]

66. Swinson, R.P. The GAD-7 scale was accurate for diagnosing generalised anxiety disorder. Evid. Based Med. 2006, 11, 184. [CrossRef]

67. Furukawa, T.A.; Katherine Shear, M.; Barlow, D.; Gorman, J.M.; Woods, S.W.; Money, R.; Etschel, E.; Engel, R.R.; Leucht, S. Evidence-based guidelines for interpretation of the Panic Disorder Severity Scale. Depress. Anxiety 2009, 26, 922-929. [CrossRef]

68. Shear, M.K.; Brown, T.A.; Barlow, D.H.; Money, R.; Sholomskas, D.E.; Woods, S.W.; Gorman, J.M.; Papp, L.A. Multicenter collaborative Panic Disorder Severity Scale. Am. J. Psychiatry 1997, 154, 1571-1575. [CrossRef]

69. Shear, M.K.; Rucci, P.; Williams, J.; Frank, E.; Grochocinski, V.; Vander Bilt, J.; Houck, P.; Wang, T. Reliability and validity of the Panic Disorder Severity Scale: Replication and extension. J. Psychiatr. Res. 2001, 35, 293-296. [CrossRef]

70. Gache, P.; Michaud, P.; Landry, U.; Accietto, C.; Arfaoui, S.; Wenger, O.; Daeppen, J.B. The Alcohol Use Disorders Identification Test (AUDIT) as a screening tool for excessive drinking in primary care: Reliability and validity of a French version. Alcohol. Clin. Exp. Res. 2005, 29, 2001-2007. [CrossRef] [PubMed]

71. Saunders, J.B.; Aasland, O.G.; Babor, T.F.; Delafuente, J.R.; Grant, M. Development of the Alcohol-Use Disorders Identification Test (Audit)—WHO Collaborative Project on Early Detection of Persons with Harmful Alcohol-Consumption 2. Addiction 1993, 88, 791-804. [CrossRef] [PubMed]

72. Cullen, F.; Link, B.; Wolfe, N.; Frank, J. The social dimensions of correctional officer stress. Justice Q. 1985, 2, 505-533. [CrossRef]

73. Paoline, E.; Lambert, H.; Hogan, N. A calm and happy keeper of the keys: The impact of ACA views, relations with coworkers, and policy views on the job stress and job satisfaction of correctional staff. Prison J. 2006, 86, 182-205. [CrossRef]

74. Ricciardelli, R.; Czarnuch, S.; Carleton, R.N.; Gacek, J.; Shewmake, J. Canadian public safety personnel and occupational stressors: How PSP interpret stressors on duty. Int. J. Environ. Res. Public Health 2020, 17, 4736. [CrossRef] [PubMed]

75. Regehr, C.; Goldberg, G.; Glancy, G.D.; Knott, T. Posttraumatic symptoms and disability in paramedics. Can. J. Psychiatry Revue Canadienne de Psychiatrie 2002, 47, 953-958. [CrossRef] [PubMed]

76. Turner, R.J.; Brown, R.L. Social support and mental health. In A Handbook for the Study of Mental Health: Social Contexts, Theories, and Systems, 2nd ed.; Scheid, T.L., Brown, T.N., Eds.; Cambridge University Press: Cambridge, UK, 2009; pp. $200-212$.

77. Denhof, M.D.; Spinaris, C.G.; Morton, G.R. Occupational Stressors in Corrections Organizations: Types, Effects and Solutions; National Institute of Corrections: Washington, DC, USA, 2014. Available online: https://info.nicic.gov/nicrp/system/files/028299.pdf (accessed on 13 August 2021).

78. Ferdik, F.; Smith, H. Correctional Officer Safety and Wellness Literature Synthesis; National Institute of Justice: Washington, DC, USA, 2017. Available online: https:/ / www.ncjrs.gov/pdffiles1/nij/250484.pdf (accessed on 13 August 2021). 